$\mathbf{T}_{\text {he }} \mathbf{J}_{\text {ournal of }} \mathbf{N}_{\text {onlinear }} \mathbf{S}_{\text {ciences and }}$ Applications

http://www.tjnsa.com

\title{
STABILITY AND STABILIZATION OF IMPULSIVE AND SWITCHED HYBRID STOCHASTIC DELAY SYSTEMS
}

\author{
JUN LIU ${ }^{1}$, XINZHI LIU ${ }^{2 \star}$, AND WEI-CHAU XIE ${ }^{3}$
}

\begin{abstract}
Stability analysis is performed and stabilization strategies are proposed for a general class of stochastic delay differential equations subjected to switching and impulses. Hybrid switching and impulses are combined to exponentially stabilize an otherwise unstable stochastic delay system. Three differential stabilization strategies are proposed, i.e. the average dwell-time approach, the impulsive stabilization, and a combined strategy. Both moment stability and almost sure stability of the resulting impulsive and switched hybrid stochastic delay systems are investigated using the well-known LyapunovRazumikhin method in the hybrid and stochastic setting. Several examples are presented to illustrate the main results and numerical simulations are presented to demonstrate the analytical results.
\end{abstract}

\section{INTRODUCTION}

In the face of growing complexity of both natural and man-made dynamical systems being encountered in various fields of applications, hybrid systems are currently becoming a large and growing interdisciplinary area of research. A hybrid system is a dynamical system that exhibits both continuous and discrete dynamic behavior. The interaction of continuous- and discrete-time dynamics in a hybrid system can lead to very rich dynamical behavior and phenomena that are not encountered in purely continuous- or discrete-time systems and hence brings difficulties and challenges to the studies of hybrid systems, such as their stability analysis and control design (see, e.g., [5, 13, 23, 24] and references therein).

Date: Received: Jun 21, 2010; Revised: December 12, 2011.

${ }^{*}$ Corresponding author Tel.: +1 519 8884567x36007; fax: +1 5197464319

(c) 2011 N.A.G.

2000 Mathematics Subject Classification. Primary 93C30; Secondary 34K35, 93E15.

Key words and phrases. Switched system, impulsive system, hybrid system, delay system, stochastic system, exponential stability, impulsive stabilization, Lyapunov-Razumikhin method. 
Impulsive differential equations or impulsive dynamical systems model real world processes that undergo abrupt changes (impulses) in the state at discrete times [10]. Particularly, impulse control and stabilization as a powerful tool to achieve stability for dynamical systems that can be highly unstable, in the absence of impulses, has gained increasing popularity and found successful applications in a wide variety of areas, such as control systems, control and synchronization of chaotic systems, complex dynamical networks, large-scale dynamical systems, secure communication, spacecraft maneuvers, population growth and biological systems, neural networks, ecosystems management, and pharmacokinetics (see [18] and references therein). Impulsive dynamical systems can be naturally viewed as a class of hybrid systems that consist of three elements: a continuous differential equation, which governs the continuous evolution of the system of between impulses; a difference equation, which governs the way the system states are changed at impulse times; and an impulsive law for determining the impulse times.

Another important type of hybrid systems are switched systems. A switched system is described by a differential equation whose right-hand side is chosen from a family of functions based on a switching signal. For each switching signal, the switched system is a time-varying differential equation. We usually study the properties of a switched system not under a particular switching signal but rather under various classes of switching signals (see, e.g., [7, 13]).

Both hybrid systems, impulsive systems and switched systems can be naturally combined to form a more comprehensive model, i.e. impulsive and switched system, in which the switching signal and the impulsive law can be integrated as an impulsive and switching law (to be described in this paper). Despite the apparent abundance of applications, impulsive and switched systems only received moderate attention since the 2000s (see, e.g., [11, 25, 26]), although, earlier in 1984, switching and impulses have already been combined to provide control for a reflected diffusion 12 .

Even though deterministic hybrid models can capture a wide range of behaviors encountered in practice, stochastic features are also very important, because of the uncertainty inherent in most applications and environmental noise ubiquitous in the real world. There has been increasing interest in stochastic hybrid systems due to their applications in areas such as insurance pricing, power industry, flexible manufacturing, fault tolerant control, maneuvering aircraft, and communication networks (see [1, 2, 21] and references therein). The theory of stochastic differential equations and stochastic processes provides necessary tools to formulate and study stochastic hybrid systems.

Most of the hybrid models currently considered in the literature use ordinary differential equations (ODEs) or its stochastic counterpart, stochastic differential equations (SDEs). The ODE and SDE models assume that the system under consideration is governed by a principle of causality, i.e the future state of the system is independent of the past states and depends only on the present state. It is well-known that the principle of causality is usually only a first approximation to the real situation and, in many applications, a more realistic model has to include some of the past states of the system, which leads to time-delay models using delay (retarded or functional) differential equations in the classical setting 
(see, e.g., [3, 4, 6, 9]) or its stochastic counterpart, stochastic delay (retarded or functional) differential equations (see, e.g., [20, 22, 9]). Incorporating time delay in the hybrid models gives rise to hybrid delay systems. Two important classes of hybrid delay systems are impulsive delay systems (or impulsive delay differential equations) and switched delay systems, which both received increasing attention in recent years (see, e.g., [14, 15, 16, 27, 28, 30]). However, including time delay makes a dynamical system infinite dimensional and hence its stability analysis more involved and challenging.

The aim of this paper is to formulate a general mathematical framework for practical systems that may exhibit all the hybrid characteristics of impulse effects, switching, stochastic dynamics, and time delays and perform stability analysis and propose stabilization strategies for such systems. It investigates stability and stabilization for a general class of impulsive and switched hybrid stochastic delay systems. Both moment stability and almost sure stability are investigated and three different stabilization strategies are proposed, i.e. the average dwell-time approach, the impulsive stabilization, and a combined strategy.

The rest of this paper is organized as follows. In Section 2, we give some necessary notations and then the general formulation of a class of impulsive and switched hybrid stochastic delay systems (ISHSDSs). The main results are presented in Section 3, in which stability analysis and stabilization strategies are proposed for the ISHSDSs formulated in Section 2. Section 3.1 deals with the the average dwell-time approach, Section 3.2 investigates the impulsive stabilization, and Section 3.3 combines both the average dwell-time approach and the impulsive stabilization strategy. In Section 4, almost sure stability results are established. Finally, in Section 5, several examples are presented and their numerical simulations are also included to demonstrate the main results.

\section{Preliminaries}

Let $\mathbb{Z}^{+}$denote the set of all positive integers, $\mathbb{R}^{n}$ the $n$-dimensional real Euclidean space, and $\mathbb{R}^{n \times m}$ the space of $n \times m$ real matrices. For $x \in \mathbb{R}^{n},|x|$ denotes the Euclidean norm of $x$. For $A=\left(a_{i j}\right) \in \mathbb{R}^{n \times m}$, define $|A|:=\sqrt{\operatorname{trace}\left(A^{T} A\right)}=$ $\sqrt{\sum_{i=1}^{n} \sum_{j=1}^{m}\left|a_{i j}\right|^{2}}$ and $\|A\|:=\sqrt{\lambda_{\max }\left(A^{T} A\right)}$, i.e. $|A|$ and $\|A\|$ denote the Frobenius norm and spectral norm of the matrix $A$, respectively.

For $-\infty<a<b<\infty$, we say that a function from $[a, b]$ to $\mathbb{R}^{n}$ is piecewise continuous, if the function has at most a finite number of jump discontinuities on $(a, b]$ and are continuous from the right for all points in $[a, b)$. Given $r>0$, $\mathcal{P C}\left([-r, 0] ; \mathbb{R}^{n}\right)$ denotes the family of piecewise continuous functions from $[-r, 0]$ to $\mathbb{R}^{n}$. A norm on $\mathcal{P C}\left([-r, 0] ; \mathbb{R}^{n}\right)$ is defined as $\|\phi\|:=\sup _{-r \leq s \leq 0}|\phi(s)|$ for $\phi \in$ $\mathcal{P C}\left([-r, 0] ; \mathbb{R}^{n}\right)$. For simplicity, $\mathcal{P C}$ is used for $\mathcal{P C}\left([-r, 0] ; \mathbb{R}^{n}\right)$ for the rest of this paper.

Let $\mathcal{N}$ be a finite index set. By a impulsive and switching law, we mean a pair $(\sigma, I)$, where $\sigma$ denotes a switching signal, which is a piecewise constant and right continuous function from $[0, \infty)$ to $\mathcal{N}$, with finitely many discontinuities on each 
bounded subinterval of $[0, \infty)$, and

$$
I: \mathcal{N} \times \mathcal{N} \times \mathbb{R}^{+} \times \mathcal{P C} \rightarrow \mathbb{R}^{n}
$$

is an impulse function (or simply an impulse). For a particular impulsive and switching law $(\sigma, I)$, let $\varpi(\sigma, I)$ be an increasing sequence $\left\{t_{k}: t_{k+1}>t_{k}, k \in \mathbb{Z}^{+}\right\}$ in $\mathbb{R}^{+}$, which includes, but not restricted to, all discontinuities (switching times) of $\sigma$. The elements in $\varpi(\sigma, I)$ are called impulse and switching times of $(\sigma, I)$. The notation $\varpi(\sigma, I)$ is used to emphasize that $t_{k}^{\prime} s$ are associated to the pair $(\sigma, I)$.

Let $(\Omega, \mathcal{F}, P)$ be a given complete probability space with $\left\{\mathcal{F}_{t}\right\}_{t \geq 0}$ as a filtration satisfying the usual conditions, and $W(t)$ be an $m$-dimensional standard Wiener process defined on $(\Omega, \mathcal{F}, P)$ and adapted to $\left\{\mathcal{F}_{t}\right\}_{t \geq 0}$. For $p>0$ and $t \geq 0$, let $\mathcal{L}_{\mathcal{F}_{t}}^{p}$ denote the family of all $\mathcal{F}_{t}$-measurable $\mathcal{P C}$-valued random variables $\phi$ such that $\mathbb{E}\left(\|\phi\|^{p}\right)<\infty$. Let $\mathcal{L}_{\mathcal{F}_{t}}^{\mathrm{b}}$ be the family of $\mathcal{P C}$-valued random variables that are bounded and $\mathcal{F}_{t}$-measurable.

Consider the impulsive switched stochastic delay system

$$
\left\{\begin{aligned}
d x(t) & =f_{\sigma}\left(t, x_{t}\right) d t+g_{\sigma}\left(t, x_{t}\right) d W(t), \quad t \notin \varpi(\sigma, I), \quad t \geq t_{0}, \\
\Delta x(t) & =I\left(\sigma\left(t^{-}\right), \sigma(t), t, x_{t^{-}}\right), \quad t \in \varpi(\sigma, I), \\
x_{t_{0}} & =\xi
\end{aligned}\right.
$$

where $x_{t}$ is defined by $x_{t}(s)=x(t+s)$, for $-r \leq s \leq 0$, and can be treated as a $\mathcal{P C}$-valued stochastic process. Similarly, the $\mathcal{P C}$-valued stochastic process $x_{t^{-}}$is defined by $x_{t^{-}}(s)=x(t+s)$, for $s \in[-r, 0)$, and $x_{t^{-}}(0)=x\left(t^{-}\right)$, where $x\left(t^{-}\right)=\lim _{s \rightarrow t^{-}} x(s)$ and $\sigma\left(t^{-}\right)=\lim _{s \rightarrow t^{-}} \sigma(s)$. For each $i \in \mathcal{N}$, both

$$
f_{i}: \mathbb{R}^{+} \times \mathcal{P C} \rightarrow \mathbb{R}^{n}
$$

and

$$
g_{i}: \mathbb{R}^{+} \times \mathcal{P C} \rightarrow \mathbb{R}^{n \times m},
$$

are Borel measurable. Moreover, $f_{i}, g_{i}$, and $I$ are assumed to satisfy necessary assumptions so that, for any initial data $\xi \in \mathcal{L}_{\mathcal{F}_{t_{0}}}^{\mathrm{b}}$ and a certain pair $(\sigma, I)$, system (2.1) has a unique global solution, denoted by $x(t ; \xi, \sigma, I)$, and, moreover, $x_{t}(\xi, \sigma, I) \in \mathcal{L}_{\mathcal{F}_{t}}^{p}$ for all $t \geq t_{0}$ and $p>0$ (e.g., see [17] for existence and uniqueness results for general impulsive and switched hybrid stochastic delay systems including (2.1) and see [20] and [22] for stochastic functional (delay) differential equations without impulse and switching). In addition, it is assumed that $f_{i}(t, 0) \equiv 0, g_{i}(t, 0) \equiv 0$, and $I(i, j, t, 0)=0$, for all $i, j \in \mathcal{N}$ and $t \in[0, \infty)$, so that system (2.1) admits a trivial solution. Note that, if $(\sigma, I)$ is given, the impulse function $I$ can also be interpreted as a sequence of functions $I_{k}$ by letting $I_{k}(\phi)=I\left(\sigma\left(t_{k}^{-}\right), \sigma\left(t_{k}\right), t_{k}, \phi\right)$, where $t_{k}^{\prime} s$ are the consecutive elements in $\varpi(\sigma, I)$. It is clear from the formulation of (2.1) that, while the switching signal $\sigma$ effectively selects the current mode of the continuous evolution, the impulse function $I$ resets the state variable at each discrete time in $\varpi(\sigma, I)$.

Definition 2.1. For $p>0$, the trivial solution of system $(2.1)$ is said to be pth moment globally uniformly exponentially stable (g.u.e.s.) on an impulsive 
and switching law set $\mathcal{I}_{\mathcal{S}}$, if for any given initial data $\xi \in \mathcal{L}_{\mathcal{F}_{t_{0}}}^{\mathrm{b}}$ and each pair $(\sigma, I) \in \mathcal{I}_{\mathcal{S}}$, the solution $x(t ; \xi, \sigma, I)$ satisfies

$$
\mathbb{E}\left(|x(t ; \xi, \sigma, I)|^{p}\right) \leq C \mathbb{E}\left(\|\xi\|^{p}\right) e^{-\varepsilon\left(t-t_{0}\right)}, \quad t \geq t_{0},
$$

where $\varepsilon$ and $C$ are positive constants independent of $t_{0}$ and the choice of $(\sigma, I)$ in $\mathcal{I}_{\mathcal{S}}$. It follows from 2.2 that

$$
\limsup _{t \rightarrow \infty} \frac{1}{t} \log \mathbb{E}\left(|x(t ; \xi, \sigma, I)|^{p}\right) \leq-\varepsilon .
$$

The left-hand side of (2.3) is called the pth moment Lyapunov exponent for the solution. Moreover, define

$$
\limsup _{t \rightarrow \infty} \frac{1}{t} \log |x(t ; \xi, \sigma, I)|
$$

to be the Lyapunov exponent of the solution. The trivial solution of system (2.1) is said to be almost surely exponentially stable if the Lyapunov exponent is almost surely negative for any $\xi \in \mathcal{L}_{\mathcal{F}_{t_{0}}}^{\mathrm{b}}$.

Remark 2.1. From Definition 2.1, one can see that the stability considered here is not only "uniform" with respect to the initial time, but also "uniform" with respect to an admissible set of impulsive and switching laws $\mathcal{I}_{\mathcal{S}}$ (see [7] for this notion of uniformity for switched linear systems with respect to a particular set of switching signals).

The following definition formulates some particular classes of impulsive and switching laws to be used in this paper. The classification is based solely on the frequency of impulse and switching times.

Definition 2.2. Let $\tau$ and $\delta$ be positive real numbers, $N_{0}$ a nonnegative integer, and $\mathcal{M}$ a certain subset of $\mathcal{N}$. Define

(i) $\mathcal{I}_{\mathcal{S}}^{\mathcal{A}}\left(\tau, N_{0} ; \mathcal{M}\right)$ to be the set of impulsive and switching laws with average dwell-time $\tau$ and chatter bound $N_{0}$ with respect to $\mathcal{M}$, i.e.

$$
\mathcal{I}_{\mathcal{S}}^{\mathcal{A}}\left(\tau, N_{0} ; \mathcal{M}\right):=\left\{(\sigma, I): N_{(\sigma, I)}(t, s ; \mathcal{M}) \leq \frac{\int_{[s, t]_{\mathcal{M}}} d s}{\tau}+N_{0}, \forall t \geq s \geq t_{0}\right\}
$$

where $[s, t]_{\mathcal{M}}=[s, t] \cap \sigma^{-1}(\mathcal{M})$ and $N_{(\sigma, I)}(t, s ; \mathcal{M})$ represents the cardinality of the set $\varpi(\sigma, I) \cap[s, t]_{\mathcal{M}}$;

(ii) $\mathcal{I}_{\mathcal{S}}^{\mathcal{U}}(\delta ; \mathcal{M})$ to be the set of impulsive and switching laws with dwell-time upper bound $\delta$ with respect to $\mathcal{M}$, i.e.

$$
\mathcal{I}_{\mathcal{S}}^{\mathcal{U}}(\delta ; \mathcal{M}):=\left\{(\sigma, I): t_{k}-t_{k+1} \leq \delta, \forall k \in \mathbb{Z}^{+} \text {s.t. } \sigma\left(t_{k-1}\right) \in \mathcal{M}\right\},
$$

where $t_{k}\left(k \in \mathbb{Z}^{+}\right)$are the consecutive members in $\varpi(\sigma, I)$ and $t_{0}$ is the initial time.

Definition 2.3. Let $\mathcal{C}^{1,2}$ denote the set of all functions from $\left[t_{0}-r, \infty\right) \times \mathbb{R}^{n}$ to $\mathbb{R}^{+}$that are continuously differentiable in $t$ and twice continuously differentiable 
in $x$. Suppose $\left\{V_{i} ; i \in \mathcal{N}\right\}$ is a family of functions in $\mathcal{C}^{1,2}$. For each $i \in \mathcal{N}$, define an operator from $\mathbb{R}^{+} \times \mathcal{P C}$ to $\mathbb{R}$ by

$\mathcal{L} V_{i}(t, \phi):=V_{t}^{i}(t, \phi(0))+V_{x}^{i}(t, \phi(0)) f_{i}(t, \phi)+\frac{1}{2} \operatorname{trace}\left[g_{i}^{T}(t, \phi) V_{x x}^{i}(t, \phi(0)) g_{i}(t, \phi)\right]$, where $V_{t}^{i}, V_{x}^{i}$, and $V_{x x}^{i}$ are functions from $\mathbb{R}^{+} \times \mathbb{R}^{n}$ defined by

$$
\begin{aligned}
V_{t}^{i}(t, x) & =\frac{\partial V_{i}(t, x)}{\partial t}, \quad V_{x}^{i}(t, x)=\left(\frac{\partial V_{i}(t, x)}{\partial x_{1}}, \cdots, \frac{\partial V_{i}(t, x)}{\partial x_{n}}\right), \\
V_{x x}^{i}(t, x) & =\left(\frac{\partial^{2} V_{i}(t, x)}{\partial x_{i} \partial x_{j}}\right)_{n \times n} .
\end{aligned}
$$

\section{Exponential Stability of the $p$ th Moment}

3.1. Average dwell-time approach. In this section, the impulses are only considered as perturbations. The sufficient conditions for exponential stability are derived in terms of average dwell-time and Razumikhin-type conditions.

Theorem 3.1. Let $p, c_{1}, c_{2}, \hat{d}, \lambda_{i}$, and $\rho_{i} \geq 1(i \in \mathcal{N})$ be positive constants. Suppose that

(i) there exists a family of functions $\left\{V_{i}: i \in \mathcal{N}\right\} \subset \mathcal{C}^{1,2}$ such that

$$
c_{1}|x|^{p} \leq V_{i}(t, x) \leq c_{2}|x|^{p},
$$

for $(i, t, x) \in \mathcal{N} \times\left[t_{0}-r, \infty\right) \times \mathbb{R}^{n}$,

(ii) there exists a family of continuous functions $\left\{\mu_{i}: i \in \mathcal{N}\right\}$ from $\left[t_{0}, \infty\right)$ to $\mathbb{R}$ such that

$$
\mathbb{E}\left(\mathcal{L} V_{i}(t, \phi)\right) \leq \mu_{i}(t) \mathbb{E}\left(V_{i}(t, \phi(0))\right),
$$

whenever $i \in \mathcal{N}, t \geq t_{0}$, and $\phi \in \mathcal{L}_{\mathcal{F}_{t}}^{p}$ satisfies

$$
\min _{i \in \mathcal{N}} \mathbb{E}\left(V_{i}(t+s, \phi(s))\right) \leq q \mathbb{E}\left(V_{i}(t, \phi(0))\right), \quad \forall s \in[-r, 0]
$$

where $q \geq e^{\mu r}$ is a finite constant and

$$
\mu=\max _{i \in \mathcal{N}} \sup _{t \in\left[t_{0}, \infty\right)} \mu_{i}^{-}(t)<\infty,
$$

where $\mu_{i}^{-}=\max \left(0,-\mu_{i}\right)$ is the negative part of the function $\mu_{i}$,

Then the trivial solution of system (2.1) is pth moment globally uniformly exponentially stable on $\mathcal{I}_{\mathcal{S}}$, where $\mathcal{I}_{\mathcal{S}}$ includes all $(\sigma, I)$ satisfying

(a) $(\sigma, I) \in \mathcal{I}_{\mathcal{S}}^{\mathcal{A}}\left(\tau_{i}, N_{0} ;\{i\}\right)$ for each $i \in \mathcal{N}$

(b) there exists a function $d:\left[t_{0}, \infty\right) \rightarrow[1, \infty)$ such that

$$
\mathbb{E}\left(V_{i}(t, \phi(0)+I(i, j, t, \phi))\right) \leq \rho_{i} d(t) \mathbb{E}\left(V_{j}(t, \phi(0))\right),
$$

for $(t, \phi) \in\left[t_{0}, \infty\right) \times \mathcal{L}_{\mathcal{F}_{t}}^{p}$ and $i, j \in \mathcal{N}$,

(c) $\int_{t_{0}}^{t}\left[\mu_{\sigma(s)}(s)+\lambda_{\sigma(s)}\right] d s \leq 0$, for all $t \geq t_{0}$,

(d) $\prod_{t_{k} \in \varpi(\sigma, I)} d\left(t_{k}\right) \leq \hat{d}$, and

(e) $\tau_{i}>\ln \left(\rho_{i}\right) / \lambda_{i}$ for each $i \in \mathcal{N}$,

and its pth moment Lyapunov exponent is guaranteed to be not greater than $-\Lambda$, where $\Lambda=\min _{i \in \mathcal{N}}\left\{\lambda_{i}-\ln \left(\rho_{i}\right) / \tau_{i}\right\}$. 
Proof. Given $(\sigma, I) \in \mathcal{I}_{\mathcal{S}}$ and any initial data $\xi \in \mathcal{L}_{\mathcal{F}_{t_{0}}}^{\mathrm{b}}$, the global solution $x(t ; \xi, \sigma, I)$ of $(2.1)$ is written as $x(t)$ in this proof. Let $\varpi(\sigma, I)=\left\{t_{k}: k \in \mathbb{Z}^{+}\right\}$ be the consecutive discontinuities of $\sigma$ as $k$ increases. Without loss of generality, assume the initial data $\xi$ is nontrivial so that $x(t)$ is not a trivial solution. Let $v(t)=\mathbb{E}\left(V_{\sigma(t)}(t, x(t))\right)$, for $t \geq t_{0}-r$, and

$$
u(t)=v(t)-\left\|v_{t_{0}}\right\| \prod_{i \in \mathcal{N}} \rho_{i}^{N_{(\sigma, I)}\left(t, t_{0} ;\{i\}\right)} \prod_{t_{0}<t_{k} \leq t} d\left(t_{k}\right) \exp \left\{\int_{t_{0}}^{t} \tilde{\mu}_{\sigma(s)}(s) d s\right\}, \quad t \geq t_{0},
$$

where $\left\|v_{t_{0}}\right\|=\max _{-r \leq s \leq 0} v\left(t_{0}+s\right)$ and $\tilde{\mu}_{i}(t)=\mu_{i}(t)+\eta$, with $\eta>0$ to be chosen later. Extend $u(t)$ to $\left[t_{0}-r, t_{0}\right)$ by letting $u(t)=v(t)-\left\|v_{t_{0}}\right\|$ for $t \in\left[t_{0}-r, t_{0}\right)$.

It is easy to see that $u(t)$ is continuous on $\left[t_{0}, t_{1}\right)$ and $u(t) \leq 0$ for $t \in\left[t_{0}-r, t_{0}\right]$. We proceed to show that $u(t) \leq 0$ for $t \in\left[t_{0}, t_{1}\right)$. Assume this is not true. Then $u(t) \geq \alpha$ for some $t \in\left[t_{0}, t_{1}\right)$ and $\alpha>0$. Let $t^{*}=\inf \left\{t \in\left[t_{0}, t_{1}\right): u(t)>\alpha\right\}$. Since $u\left(t_{0}\right) \leq 0$, one must have $t^{*} \in\left(t_{0}, t_{1}\right)$ and $u\left(t^{*}\right)=\alpha$. Moreover, $u(t) \leq \alpha$ for $t \in\left[t_{0}-r, t^{*}\right]$. Now for any $s \in[-r, 0]$, one has

$$
\begin{aligned}
v\left(t^{*}+s\right) & \leq \alpha+\left\|v_{t_{0}}\right\| \exp \left(\int_{t_{0}}^{t^{*}+s} \tilde{\mu}_{\sigma(\theta)}(\theta) d \theta\right) \\
& \leq \max \left\{1, \exp \left(\int_{t^{*}}^{t^{*}+s} \tilde{\mu}_{\sigma(\theta)}(\theta) d \theta\right)\right\} v\left(t^{*}\right) \\
& \leq q v\left(t^{*}\right),
\end{aligned}
$$

since

$$
\int_{t^{*}}^{t^{*}+s} \tilde{\mu}_{\sigma(\theta)}(\theta) d \theta \leq \int_{t^{*}}^{t^{*}+s} \mu_{\sigma(\theta)}(\theta) d \theta \leq \int_{t^{*}+s}^{t^{*}} \mu_{\sigma(\theta)}^{-}(\theta) d \theta \leq \mu r .
$$

By $(3.2)$ and 3.3$)$, one has

$$
\mathbb{E}\left(\mathcal{L} V_{\sigma\left(t^{*}\right)}\left(t^{*}, x_{t^{*}}\right)\right) \leq \mu_{\sigma\left(t^{*}\right)}\left(t^{*}\right) \mathbb{E}\left(V_{\sigma\left(t^{*}\right)}\left(t^{*}, x\left(t^{*}\right)\right)\right) .
$$

Since $u\left(t^{*}\right)=\alpha>0$, it is clear that $\mathbb{E}\left(V_{\sigma\left(t^{*}\right)}\left(t^{*}, x\left(t^{*}\right)\right)\right)=v\left(t^{*}\right)>0$. Hence

$$
\begin{aligned}
& \mathbb{E}\left(\mathcal{L} V_{\sigma\left(t^{*}\right)}\left(t^{*}, x_{t^{*}}\right)\right)-\tilde{\mu}_{\sigma\left(t^{*}\right)}\left(t^{*}\right) \mathbb{E}\left(V_{\sigma\left(t^{*}\right)}\left(t^{*}, x\left(t^{*}\right)\right)\right) \\
& \quad<\mathbb{E}\left(\mathcal{L} V_{\sigma\left(t^{*}\right)}\left(t^{*}, x_{t^{*}}\right)\right)-\mu_{\sigma\left(t^{*}\right)}\left(t^{*}\right) \mathbb{E}\left(V_{\sigma\left(t^{*}\right)}\left(t^{*}, x\left(t^{*}\right)\right)\right) \\
& \quad \leq 0,
\end{aligned}
$$

which, by continuity, implies that

$$
\mathbb{E}\left(\mathcal{L} V_{\sigma(\theta)}\left(\theta, x_{\theta}\right)\right)-\tilde{\mu}_{\sigma(\theta)}(\theta) \mathbb{E}\left(V_{\sigma(\theta)}(\theta, x(\theta))\right) \leq 0, \quad \theta \in\left[t^{*}, t^{*}+h\right],
$$

provided that $h$ is sufficiently small. Applying Itô's formula on $\left[t^{*}, t^{*}+h^{\prime}\right]$, where $h^{\prime} \in[0, h]$, one has

$$
\begin{aligned}
u\left(t^{*}+h^{\prime}\right)-u\left(t^{*}\right) & =\int_{t^{*}}^{t^{*}+h^{\prime}}\left[\mathbb{E}\left(\mathcal{L} V_{\sigma(\theta)}\left(\theta, x_{\theta}\right)\right)-\tilde{\mu}_{\sigma(\theta)}(\theta) \mathbb{E}\left(V_{\sigma(\theta)}(\theta, x(\theta))\right)\right] d \theta \\
& \leq 0
\end{aligned}
$$

for all $h^{\prime} \in[0, h]$, which contradicts the definition of $t^{*}$. Therefore, one must have $u(t) \leq 0$ for $t \in\left[t_{0}, t_{1}\right)$. 
Now assume that $u(t) \leq 0, \quad \forall t \in\left[t_{0}-r, t_{m}\right)$, where $m \geq 1$ is a positive integer. We proceed to show that $u(t) \leq 0$ on $\left[t_{m}, t_{m+1}\right)$. To derive a contradiction, assume that $u(t) \leq 0$ does not hold on $\left[t_{m}, t_{m+1}\right)$. Then $u(t) \geq \alpha$ for some $t \in\left[t_{m}, t_{m+1}\right)$ and some $\alpha>0$. Let $t^{*}=\inf \left\{t \in\left[t_{m}, t_{m+1}\right): u(t)>\alpha\right\}$. Since, by (3.5),

$$
\begin{aligned}
u\left(t_{m}\right) & =v\left(t_{m}\right)-\left\|v_{t_{0}}\right\| \prod_{i \in \mathcal{N}} \rho_{i}^{N_{(\sigma, I)}\left(t_{m}, t_{0} ;\{i\}\right)} \prod_{t_{0}<t_{k} \leq t_{m}} d\left(t_{k}\right) \exp \left(\int_{t_{0}}^{t_{m}} \tilde{\mu}_{\sigma(\theta)}(\theta) d \theta\right) \\
\leq & \rho_{\sigma\left(t_{m}\right)} d\left(t_{m}\right)\left\{v\left(t_{m}^{-}\right)-\left\|v_{t_{0}}\right\| \prod_{i \in \mathcal{N}} \rho_{i}^{N_{(\sigma, I)}\left(t_{m}^{-}, t_{0} ;\{i\}\right)}\right. \\
& \left.\times \prod_{t_{0}<t_{k}<t_{m}} d\left(t_{k}\right) \exp \left(\int_{t_{0}}^{t_{m}} \tilde{\mu}_{\sigma(\theta)}(\theta) d \theta\right)\right\} \\
\leq 0 &
\end{aligned}
$$

one must have $t^{*} \in\left(t_{m}, t_{m+1}\right)$ and $u\left(t^{*}\right)=\alpha$. Moreover, $u(t) \leq \alpha$ for $t \in\left[t_{0}-r, t^{*}\right]$. For $s \in[-r, 0]$, one now has,

$$
\begin{aligned}
v\left(t^{*}+s\right) & \leq \alpha+\left\|v_{t_{0}}\right\| \prod_{i \in \mathcal{N}} \rho_{i}^{N_{(\sigma, I)}\left(t^{*}+s, t_{0} ;\{i\}\right)} \prod_{t_{0}<t_{k} \leq t^{*}+s} d\left(t_{k}\right) \exp \left(\int_{t_{0}}^{t^{*}+s} \tilde{\mu}_{\sigma(\theta)}(\theta) d \theta\right) \\
& \leq \max \left\{1, \exp \left(\int_{t^{*}}^{t^{*}+s} \tilde{\mu}_{\sigma(\theta)}(\theta) d \theta\right)\right\} v\left(t^{*}\right) \\
& \leq q v\left(t^{*}\right) .
\end{aligned}
$$

Repeating the same argument as on $\left[t_{0}, t_{1}\right)$, one can derive a contradiction and hence show that $u(t) \leq 0$ for $t \in\left[t_{m}, t_{m+1}\right)$. By induction on $m$, one can conclude that $u(t) \leq 0$ for all $t \geq t_{0}$, which implies

$$
v(t) \leq \hat{d}\left\|v_{t_{0}}\right\| \prod_{i \in \mathcal{N}} \rho_{i}^{N_{(\sigma, I)}\left(t, t_{0} ;\{i\}\right)} \exp \left(\int_{t_{0}}^{t} \tilde{\mu}_{\sigma(s)}(s)\right) d s, \quad \forall t \geq t_{0} .
$$

Since $\eta>0$ is arbitrary and independent of $t,(3.6)$ actually implies

$$
v(t) \leq \hat{d}\left\|v_{t_{0}}\right\| \prod_{i \in \mathcal{N}} \rho_{i}^{N_{(\sigma, I)}\left(t, t_{0} ;\{i\}\right)} \exp \left(\int_{t_{0}}^{t} \mu_{\sigma(s)}(s) d s\right), \quad \forall t \geq t_{0} .
$$

By (3.1) and the fact that $\left.(\sigma, I) \in \mathcal{I}_{\mathcal{S}}, 3.7\right)$ gives

$$
\begin{aligned}
\mathbb{E}\left(|x(t)|^{p}\right) & \leq C \mathbb{E}\left(\|\xi\|^{p}\right) \exp \left\{\sum_{i \in \mathcal{N}} \int_{\left[t_{0}, t\right] \cap \sigma^{-1}(\{i\})} \frac{\ln \left(\rho_{i}\right)}{\tau_{i}} d s-\int_{t_{0}}^{t} \lambda_{\sigma(s)} d s\right\} \\
& \leq C \mathbb{E}\left(\|\xi\|^{p}\right) \exp \left\{-\int_{t_{0}}^{t}\left[\lambda_{\sigma(s)}-\frac{\ln \left(\rho_{\sigma(s)}\right)}{\tau_{\sigma(s)}}\right] d s\right\} \\
& \leq C \mathbb{E}\left(\|\xi\|^{p}\right) e^{-\Lambda\left(t-t_{0}\right)}
\end{aligned}
$$

where $C=\hat{d} \prod_{i \in \mathcal{N}} \rho_{i}^{N_{0}} c_{2} / c_{1}$, which shows that the $p$ th moment of system 2.1 is g.u.e.s with its $p$ th moment Lyapunov exponent not greater than $-\Lambda$. 
Remark 3.1. The formulation of the set $\mathcal{I}_{\mathcal{S}}$ of impulsive and switching laws in Theorem 3.1 essentially proposes an average dwell-time condition $\tau_{i}>\ln \left(\rho_{i}\right) / \lambda_{i}$ for each individual mode, which generalizes the well-known average dwell-time notion from [8]. Moreover, it allows the existence of unstable mode (i.e. when $\mu_{i}(t)$ might be positive on certain subintervals of $\mathbb{R}^{+}$), as long as the switching between the stable modes and unstable modes results a "stable" balance such that $\int_{t_{0}}^{t}\left[\mu_{\sigma(s)}(s)+\lambda_{\sigma(s)}\right] d s \leq 0$ (see, e.g., 29] for results on stability of switched system with both stable and unstable modes).

Remark 3.2. According to Theorem 3.1, the average dwell-time condition for the $i$ th mode is given by $\tau_{i}>\ln \left(\rho_{i}\right) / \lambda_{i}$. If $\rho_{i}=1$, this condition reduces to $\tau_{i}>0$, which would read as the $i$ th mode has a positive average dwell-time condition. However, a closer scrutiny of the proof (see the estimate (3.7)) reveals that there is essentially no average dwell-time restriction on the $i$ th mode, if $(3.5)$ is satisfied with $\rho_{i}=1$.

3.2. Impulsive stabilization. In this section, assuming that all the subsystems are unstable, impulses are added as a stabilizing mechanism to exponentially stabilize system (2.1).

Theorem 3.2. Let $\Lambda, p, c_{1}, c_{2}, \rho_{i}<1, \delta_{i}(i \in \mathcal{N}), \hat{d}$ be positive constants and $\bar{\nu}_{i}(i \in \mathcal{N})$ be nonnegative numbers. Suppose that

(i) condition (i) of Theorem 3.1 holds,

(ii) there exists a family of continuous functions $\left\{\nu_{i}: i \in \mathcal{N}\right\}$ from $\left[t_{0}, \infty\right)$ to $\mathbb{R}^{+}$satisfying

$$
\sup _{t \in\left[t_{0}, \infty\right)} \int_{t}^{t+\delta_{i}} \nu_{i}(s) \leq \bar{\nu}_{i} \delta_{i}
$$

such that

$$
\mathbb{E}\left(\mathcal{L} V_{i}(t, \phi)\right) \leq \nu_{i}(t) \mathbb{E}\left(V_{i}(t, \phi(0))\right),
$$

whenever $i \in \mathcal{N}, t \geq t_{0}$, and $\phi \in \mathcal{L}_{\mathcal{F}_{t}}^{p}$ satisfies

$$
\min _{i \in \mathcal{N}} \mathbb{E}\left(V_{i}(t+s, \phi(s))\right) \leq q_{i} \mathbb{E}\left(V_{i}(t, \phi(0))\right), \quad \forall s \in[-r, 0],
$$

where $q_{i} \geq 1$ is a constant such that

$$
q_{i} \geq \exp \left(\Lambda r+\Lambda \delta_{i}+\bar{\nu}_{i} \delta_{i}\right)
$$

Then the trivial solution of system (2.1) is pth moment globally uniformly exponentially stable on $\mathcal{I}_{\mathcal{S}}$, where $\mathcal{I}_{\mathcal{S}}$ includes all $(\sigma, I)$ satisfying

(a) $(\sigma, I) \in \mathcal{S}\left(\delta_{i} ;\{i\}\right)$ for all $i \in \mathcal{N}$,

(b) same as (b) in Theorem 3.1.

(c) $\prod_{t_{k} \in \varpi(\sigma, I)} d\left(t_{k}\right) \leq \hat{d}$,

(d) $\delta_{i}<-\ln \left(\rho_{i}\right) /\left(\Lambda+\bar{\nu}_{i}\right)$, for all $i \in \mathcal{N}$,

and its pth moment Lyapunov exponent is not greater than $-\Lambda$.

Proof. Given $(\sigma, I) \in \mathcal{I}_{\mathcal{S}}$ and any initial data $\xi \in \mathcal{L}_{\mathcal{F}_{t_{0}}}^{\mathrm{b}}$, keep the same notation for $x(t), v(t), \varpi(\sigma, I)$ as in the proof for Theorem 3.1. Let $\tilde{\Lambda}=\Lambda-\eta$, where $\eta>0$ 
being an arbitrary number such that $\tilde{\Lambda}>0$. Choose $M \in\left(e^{\left(\Lambda+\bar{\nu}_{i_{0}}\right) \delta_{i_{0}}}, q_{i_{0}} e^{\Lambda \delta_{i_{0}}}\right)$ so that

$$
\left\|v_{t_{0}}\right\|<M\left\|v_{t_{0}}\right\| e^{-\left(\Lambda+\bar{\nu}_{i_{0}}\right) \delta_{i_{0}}}<M\left\|v_{t_{0}}\right\| e^{-\Lambda \delta_{i_{0}}}<q_{i_{0}}\left\|v_{t_{0}}\right\|
$$

where $i_{0}=\sigma\left(t_{0}\right)$ and $\left\|v_{t_{0}}\right\|=\max _{-r \leq s \leq 0} v\left(t_{0}+s\right)$. We will show that

$$
v(t) \leq M \mathbb{E}\left(\|\xi\|^{p}\right) e^{-\tilde{\Lambda}\left(t_{1}-t_{0}\right)}, \quad \forall t \in\left[t_{0}, t_{1}\right),
$$

by proving a stronger claim:

$$
v(t) \leq M\left\|v_{t_{0}}\right\| e^{-\Lambda \delta_{i_{0}}}, \quad \forall t \in\left[t_{0}, t_{1}\right) .
$$

Suppose (3.14) is not true and observe that

$$
v(t) \leq\left\|v_{t_{0}}\right\|<M\left\|v_{t_{0}}\right\| e^{-\Lambda \delta_{i_{0}}}
$$

holds on $\left[t_{0}-r, t_{0}\right]$. Define $t^{*}=\inf \left\{t \in\left[t_{0}, t_{1}\right): v(t)>M\left\|v_{t_{0}}\right\| e^{-\Lambda \delta_{i_{0}}}\right\}$. Then $t^{*} \in\left(t_{0}, t_{1}\right)$ and, by continuity of $v(t)$,

$$
v(t) \leq v\left(t^{*}\right)=M\left\|v_{t_{0}}\right\| e^{-\Lambda \delta_{i_{0}}}, \quad \forall t \in\left[t_{0}, t^{*}\right] .
$$

In view of (3.15), define $t_{*}=\sup \left\{t \in\left[t_{0}, t^{*}\right): v(t) \leq\left\|v_{t_{0}}\right\|\right\}$. Then $t_{*} \in\left[t_{0}, t^{*}\right)$ and, by continuity of $v(t)$,

$$
v(t) \geq v\left(t_{*}\right)=\left\|v_{t_{0}}\right\|, \quad \forall t \in\left[t_{*}, t^{*}\right] .
$$

Now in view of (3.12), (3.16), and (3.17), one has, for $t \in\left[t_{*}, t^{*}\right]$ and $s \in[-r, 0]$,

$$
v(t+s) \leq v\left(t^{*}\right)=M\left\|v_{t_{0}}\right\| e^{-\Lambda \delta_{i_{0}}}<q_{i_{0}}\left\|v_{t_{0}}\right\| \leq q_{i_{0}} v(t) .
$$

By the Razumikhin-type conditions (3.9) and (3.10), one has

$$
\mathbb{E}\left(\mathcal{L} V_{\sigma(t)}\left(t, x_{t}\right)\right) \leq \nu_{\sigma(t)}(t) \mathbb{E}\left(V_{\sigma(t, x(t))}\right), \quad \forall t \in\left[t_{*}, t^{*}\right] .
$$

Applying Itô's fomula on $\left[t_{*}, t^{*}\right]$ and by $(3.18)$, one obtains that

$$
\begin{aligned}
& e^{\int_{t_{0}}^{t^{*}} \nu_{\sigma(s)}(s) d s} v\left(t^{*}\right)-e^{\int_{t_{0}}^{t_{*}} \nu_{\sigma(s)}(s) d s} v\left(t_{*}\right) \\
& \quad=\int_{t_{*}}^{t^{*}} e^{\int_{t_{0}}^{s} \nu_{\sigma(s)}(s) d s}\left[\mathbb{E}\left(\mathcal{L} V_{\sigma(s)}\left(s, x_{s}\right)\right)-\nu_{\sigma(s)}(s) \mathbb{E}\left(V_{\sigma(s)}(s, x(s))\right)\right] d s \\
& \quad \leq 0
\end{aligned}
$$

which implies, by 3.8 ,

$$
v\left(t^{*}\right) \leq v\left(t_{*}\right) e^{\int_{t_{*}}^{t^{*}} \nu_{\sigma(s)}(s) d s} \leq v\left(t_{*}\right) e^{\bar{\nu}_{i_{0}} \delta_{i_{0}}} .
$$

Since 3.19 contradicts what is implied by (3.12), (3.16), and (3.17), claim (3.14) must be true and so is $(3.13)$. Although the choice of $M$ in 3.12 depends on $i_{0}$, one can choose some $M$ independent of $i_{0}$ such that 3.13 holds, due to the fact that $\mathcal{N}$ is a finite set.

Now, assume that

$$
v(t) \leq M_{k}\left\|v_{t_{0}}\right\| e^{-\tilde{\Lambda}\left(t_{k}-t_{0}\right)}, \quad \forall t \in\left[t_{k-1}, t_{k}\right)
$$

for all $k \leq m$, where $k, m \in \mathbb{Z}^{+}$and $M_{k}$ is defined by $M_{1}=M$ and $M_{k}=$ $M_{k-1} d\left(t_{k-1}\right)$, for $k \geq 2$. We proceed to show that

$$
v(t) \leq M_{m+1}\left\|v_{t_{0}}\right\| e^{-\tilde{\Lambda}\left(t_{m+1}-t_{0}\right)}, \quad \forall t \in\left[t_{m}, t_{m+1}\right),
$$


by proving a stronger claim:

$$
v(t) \leq M_{m+1}\left\|v_{t_{0}}\right\| e^{-\tilde{\Lambda}\left[\left(t_{m}-t_{0}\right)+\delta_{i_{m}}\right]}, \quad \forall t \in\left[t_{m}, t_{m+1}\right),
$$

where $i_{m}=\sigma\left(t_{m}\right)$. From 3.20 and (3.5), one has

$$
v\left(t_{m}\right) \leq \rho_{i_{m}} d\left(t_{m}\right) v\left(t_{m}^{-}\right) \leq \rho_{i_{m}} d\left(t_{m}\right) M_{m}\left\|v_{t_{0}}\right\| e^{-\tilde{\Lambda}\left(t_{m}-t_{0}\right)}=\rho_{i_{m}} M_{m+1}\left\|v_{t_{0}}\right\| e^{-\tilde{\Lambda}\left(t_{m}-t_{0}\right)},
$$

which implies, by the fact that $\sigma \in \mathcal{I}_{\mathcal{S}}$,

$$
v\left(t_{m}\right)<e^{-\bar{\nu}_{i_{m}} \delta_{i_{m}}} M_{m+1}\left\|v_{t_{0}}\right\| e^{-\tilde{\Lambda}\left[\left(t_{m}-t_{0}\right)+\delta_{i_{m}}\right]} .
$$

Choose $\varepsilon>0$ sufficiently small such that

$$
\begin{aligned}
v\left(t_{m}\right) & <e^{-\bar{\nu}_{i_{m}} \delta_{i_{m}}-\varepsilon} M_{m+1}\left\|v_{t_{0}}\right\| e^{-\tilde{\Lambda}\left[\left(t_{m}-t_{0}\right)+\delta_{i_{m}}\right]} \\
& <e^{-\bar{\nu}_{i_{m}} \delta_{i_{m}}} M_{m+1}\left\|v_{t_{0}}\right\| e^{-\tilde{\Lambda}\left[\left(t_{m}-t_{0}\right)+\delta_{i_{m}}\right]}
\end{aligned}
$$

Suppose claim (3.22) is not true. Define

$$
\bar{t}=\inf \left\{t \in\left[t_{m}, t_{m+1}\right): v(t)>M_{m+1}\left\|v_{t_{0}}\right\| e^{-\tilde{\Lambda}\left[\left(t_{m}-t_{0}\right)+\delta_{i_{m}}\right]}\right\} .
$$

In view of 3.23$)$, one has $\bar{t} \in\left(t_{m}, t_{m+1}\right)$ and, by continuity of $v(t)$.

$$
v(t) \leq v(\bar{t})=M_{m+1}\left\|v_{t_{0}}\right\| e^{-\tilde{\Lambda}\left[\left(t_{m}-t_{0}\right)+\delta_{i_{m}}\right]}, \quad \forall t \in\left[t_{m}, \bar{t}\right] .
$$

In view of 3.24 , define

$$
\underline{t}=\sup \left\{t \in\left[t_{m}, \bar{t}\right): v(t) \leq e^{-\bar{\nu}_{i_{m}} \delta_{i_{m}}-\varepsilon} M_{m+1}\left\|v_{t_{0}}\right\| e^{-\tilde{\Lambda}\left[\left(t_{m}-t_{0}\right)+\delta_{i_{m}}\right]}\right\} .
$$

Then $\underline{t} \in\left(t_{m}, \bar{t}\right)$ and, by continuity of $v(t)$,

$$
v(t) \geq v(\underline{t})=e^{-\bar{\nu}_{i_{m}} \delta_{i_{m}}-\varepsilon} M_{m+1}\left\|v_{t_{0}}\right\| e^{-\tilde{\Lambda}\left[\left(t_{m}-t_{0}\right)+\delta_{i_{m}}\right]}=e^{-\bar{\nu}_{i_{m}} \delta_{i_{m}}-\varepsilon} v(\bar{t}),
$$

for all $t \in[\underline{t}, \bar{t}]$. Now for $t \in[\underline{t}, \bar{t}]$ and $s \in[-r, 0]$, from (3.11), (3.20), (3.26), and the fact that $t+s \in\left[t_{m-1}, \bar{t}\right]$, one has

$$
\begin{aligned}
v(t+s) & \leq M_{m+1}\left\|v_{t_{0}}\right\| e^{-\tilde{\Lambda}\left(t+s-t_{0}\right)}=e^{\tilde{\Lambda}\left(t_{m}-t\right)-\tilde{\Lambda} s+\tilde{\Lambda} \delta_{i_{m}}} v(\bar{t}) \\
& \leq e^{\tilde{\Lambda} r+\tilde{\Lambda} \delta_{i_{m}}+\bar{\nu}_{i_{m}} \delta_{i_{m}}+\varepsilon} v(\underline{t}) \\
& \leq q_{i_{m}} v(t),
\end{aligned}
$$

provided that $\varepsilon$ defined in 3.24 is chosen sufficiently small. Similar to the argument on $\left[t_{*}, t^{*}\right]$, an application of Itô's formula on $[\underline{t}, \bar{t}]$ will lead to $v(\bar{t}) \leq$ $v(\underline{t}) e^{\bar{\nu}_{i_{m}} \delta_{i_{m}}}$, which would contradict $(3.26)$. Therefore, claim $(3.22)$ must be true and so is (3.21). By induction on $m$ and the definition of $M_{m}$, one can conclude that

$$
v(t) \leq M \prod_{t_{0}<t_{k} \leq t} d\left(t_{k}\right)\left\|v_{t_{0}}\right\| e^{-\tilde{\Lambda}\left(t-t_{0}\right)}, \quad \forall t \geq t_{0} .
$$

By (3.1) and the condition on $d(t)$, one has

$$
\mathbb{E}\left(|x(t)|^{p}\right) \leq M \hat{d} \frac{c_{2}}{c_{1}} \mathbb{E}\left(\|\xi\|^{p}\right) e^{-\tilde{\Lambda}\left(t-t_{0}\right)}, \quad \forall t \geq t_{0}
$$

Since $\eta>0$ is arbitrary and indpendent of $t$, we actually have shown

$$
\mathbb{E}\left(|x(t)|^{p}\right) \leq M \hat{d} \frac{c_{2}}{c_{1}} \mathbb{E}\left(\|\xi\|^{p}\right) e^{-\Lambda\left(t-t_{0}\right)}, \quad \forall t \geq t_{0}
$$


which shows that the $p$ th moment of system (2.1) is g.u.e.s with its $p$ th moment Lyapunov exponent not greater than $-\Lambda$.

3.3. Combination of average dwell-time approach and impulsive stabilization. Theorems 3.1 and 3.2 address the average dwell-time approach and impulsive stabilization, respectively. While Theorem 3.1 applies to the situation when both stable and unstable modes are present in the switched system and stability is achieved by restricting the switching signal so that a certain balance between the activation time of the stable mode and the unstable mode, Theorem 3.2 assumes all the modes are unstable and addresses how one can seek an impulsive stabilization rule for a certain class of switching signals. In this section, these two approaches are combined to achieve a stabilization result for impulsive switched stochastic delay systems.

The motivation for proposing this strategy is the fact that the impulse stabilization should be kept to a minimal in many applications due to its cost. We consider applying impulses only when the system is about to switch to an unstable mode. Otherwise, the requirement for stability is guaranteed by imposing an average dwell-time condition. For this purpose, we propose a partition of the subsystems, i.e. assume $\mathcal{N}=\mathcal{N}_{\text {avg }} \cup \mathcal{N}_{\text {imp }}$ and $\mathcal{N}_{\text {avg }} \cap \mathcal{N}_{\text {imp }}=\emptyset$. Let $\sigma[s, t]$ $\left(t \geq s \geq t_{0}\right)$ denotes the image of the interval $[s, t]$ under the mapping of $\sigma$. The combined hybrid strategy can be described as follows:

(i) on $[s, t]$ such that $\sigma[s, t] \subset \mathcal{N}_{\text {avg }}$, stabilization is achieved by an average dwell-time approach and impulsive stabilization is not performed; and

(ii) on $[s, t]$ such that $\sigma[s, t] \subset \mathcal{N}_{\text {imp }}$, an stabilizing impulse is added at each switching time between $s$ and $t$.

For $t \geq s \geq t_{0}$, let $[s, t]_{\mathrm{avg}}^{\sigma}=[s, t] \cap \sigma^{-1}\left(\mathcal{N}_{\mathrm{avg}}\right)$ and $[s, t]_{\mathrm{imp}}^{\sigma}=[s, t] \cap \sigma^{-1}\left(\mathcal{N}_{\mathrm{imp}}\right)$.

Theorem 3.3. Let $\lambda, \Lambda, p, c_{1}, c_{2}, \hat{d}, \rho_{i} \geq 1\left(i \in \mathcal{N}_{\text {avg }}\right), \tau_{i}, \delta_{i}, \rho_{i}<1$ be positive constants and $\bar{\nu}_{i}\left(i \in \mathcal{N}_{\text {imp }}\right)$ be nonnegative constants. Suppose that

(i) condition (i) of Theorem 3.1 holds,

(ii) there exists a family of continuous functions $\left\{\mu_{i}: i \in \mathcal{N}_{\text {avg }}\right\}$ from $\left[t_{0}, \infty\right)$ to $\mathbb{R}$ such that condition (ii) of Theorem 3.1 holds, for all $i \in \mathcal{N}_{\text {avg }}$, with

$$
\mu=\max _{i \in \mathcal{N}_{\text {avg }}} \sup _{t \in\left[t_{0}, \infty\right)} \mu_{i}^{-}(t),
$$

(iii) there exists a family of continuous functions $\left\{\nu_{i}: i \in \mathcal{N}_{\text {imp }}\right\}$ from $\left[t_{0}, \infty\right)$ to $\mathbb{R}^{+}$satisfying (3.8) such that condition (ii) of Theorem 3.2 is satisfied, for all $i \in \mathcal{N}_{\text {imp }}$, with

$$
q_{i} \geq \exp \left(\mu r+\Lambda \delta_{i}+\bar{\nu}_{i} \delta_{i}\right)
$$

Then the trivial solution of system (2.1) is pth moment globally uniformly exponentially stable on $\mathcal{I}_{\mathcal{S}}$, where $\mathcal{I}_{\mathcal{S}}$ includes all $(\sigma, I)$ satisfying

(a) $(\sigma, I) \in \mathcal{I}_{\mathcal{S}}^{\mathcal{A}}\left(\tau_{i}, N_{0} ;\{i\}\right)$ for all $i \in \mathcal{N}_{\text {avg }}$ and $(\sigma, I) \in \mathcal{I}_{\mathcal{S}}^{\mathcal{U}}\left(\delta_{i} ;\{i\}\right)$ for all $i \in$ $\mathcal{N}_{\text {imp }}$

(b) same as (b) in Theorem 3.1.

(c) $\prod_{t_{k} \in \varpi(\sigma, I)} d\left(t_{k}\right) \leq \hat{d}$,

(d) $\int_{\left[t_{0}, t\right]_{\text {avg }}^{\sigma}}\left[\mu_{\sigma(s)}(s)+\lambda_{\sigma(s)}\right] d s \leq 0$, for all $t \geq t_{0}$, 
(e) $\tau_{i}>\ln \left(\rho_{i}\right) / \lambda_{i}$, for all $i \in \mathcal{N}_{\text {avg }}$,

(f) $\delta_{i}<-\ln \left(\rho_{i}\right) /\left(\Lambda+\bar{\nu}_{i}\right)$, for all $i \in \mathcal{N}_{\text {imp }}$,

where $\Lambda=\min _{i \in \mathcal{N}_{\text {avg }}}\left\{\lambda_{i}-\ln \left(\rho_{i}\right) / \tau_{i}\right\}$, and its pth moment Lyapunov exponent is not greater than $-\Lambda$.

Proof. Given $(\sigma, I) \in \mathcal{I}_{\mathcal{S}}$ and any initial data $\xi \in \mathcal{L}_{\mathcal{F}_{t}}^{\mathrm{b}}$, keep the same notation for $x(t), v(t)$, and $\varpi(\sigma, I)$ as in the proof for Theorem 3.1. The proof is essentially a combination of the proofs for Theorem 3.1 and Theorem 3.2. Let $\eta \in(0, \Lambda)$ be an arbitrary number and define

$$
\tilde{\Lambda}=\Lambda-\eta, \quad \tilde{\mu}_{i}(t)=\mu_{i}(t)+\eta, \quad t \geq t_{0}, \quad i \in \mathcal{N}_{\text {avg }} .
$$

We claim that, for all $t \geq t_{0}$,

$v(t) \leq M\left\|v_{t_{0}}\right\| \prod_{i \in \mathcal{N}_{\text {avg }}} \rho_{i}^{N_{\sigma}\left(t, t_{0} ;\{i\}\right)} \prod_{t_{0}<t_{k} \leq t} d\left(t_{k}\right) \exp \left(\int_{\left[t_{0}, t\right]_{\mathrm{avg}}^{\sigma}} \tilde{\mu}_{\sigma(s)}(s) d s-\int_{\left[t_{0}, t_{\sigma}\right]_{\mathrm{imp}}^{\sigma}} \tilde{\Lambda} d s\right)$,

where $\left\|v_{t_{0}}\right\|=\max _{-r \leq s \leq 0} v\left(t_{0}+s\right), M>1$ is as chosen in the proof of Theorem 3.2. and $t_{\sigma}$ is the next switching instant of $\sigma$ beyond $t$. For simplicity, let $u(t)$ denote the right-hand side of the claimed inequality. We shall prove the claim by induction on $\left[t_{k-1}, t_{k}\right), k \in \mathbb{Z}^{+}$. Starting on $\left[t_{0}, t_{1}\right)$, consider two cases: (i) $i_{0}=\sigma\left(t_{0}\right) \in \mathcal{N}_{\text {avg }}$, (ii) $i_{0} \in \mathcal{N}_{\text {imp. }}$. We have

(i) following the argument in the proof of Theorem 3.1.

$$
v(t) \leq\left\|v_{t_{0}}\right\| e^{\int_{t_{0}}^{t} \tilde{\mu}_{\sigma(s)}(s) d s}, \quad t \in\left[t_{0}, t_{1}\right),
$$

which implies the claim on $\left[t_{0}, t_{1}\right)$,

(ii) following the argument in the proof for Theorem 3.2 .

$$
v(t) \leq M\left\|v_{t_{0}}\right\| e^{-\tilde{\Lambda} \delta_{i_{0}}}, \quad \forall t \in\left[t_{0}, t_{1}\right),
$$

which also implies the claim on $\left[t_{0}, t_{1}\right)$.

Now suppose that the claim is true on $\left[t_{0}, t_{m}\right)$, where $m \in \mathbb{Z}^{+}$. We shall show that it is also true on $\left[t_{m}, t_{m+1}\right)$. Consider the following three cases:

(a) $\sigma\left(t_{m}\right) \in \mathcal{N}_{\text {avg. }}$. Suppose that the claim is not true on $\left[t_{m}, t_{m+1}\right)$. Then there exists some $\alpha>0$ such that $v(t) \leq u(t)+\alpha$, for some $t \in\left[t_{m}, t_{m+1}\right)$. Let $t^{*}=\inf \left\{t \in\left[t_{m}, t_{m+1}\right): v(t)>u(t)+\alpha\right\}$. Since $v\left(t_{m}\right) \leq u\left(t_{m}\right)$, one must have $t^{*} \in\left(t_{m}, t_{m+1}\right)$. Moreover, by continuity of $v$ and $u$ on $\left[t_{m}, t_{m}+1\right), v\left(t^{*}\right)=u\left(t^{*}\right)+\alpha$ and $v(t) \leq u(t)+\alpha$ for $t \in\left[t_{0}-r, t^{*}\right]$. Now for any $s \in[-r, 0]$, one has

$$
v\left(t^{*}+s\right) \leq u\left(t^{*}+s\right)+\alpha \leq \max \left\{1, \frac{u\left(t^{*}+s\right)}{u\left(t^{*}\right)}\right\} v\left(t^{*}\right),
$$


where

$$
\begin{aligned}
\frac{u\left(t^{*}+s\right)}{u\left(t^{*}\right)} & =\exp \left(\int_{\left[t^{*}, t^{*}+s\right]_{\text {avg }}^{\sigma}} \tilde{\mu}_{\sigma(s)}(s) d s-\int_{\left[t_{\sigma}^{*},\left(t^{*}+s\right)_{\sigma}\right]_{\mathrm{imp}}^{\sigma}} \tilde{\Lambda} d s\right) \\
& \leq \exp \left(\int_{\left[t^{*}, t^{*}+s\right]_{\mathrm{avg}}^{\sigma}} \tilde{\mu}_{\sigma(s)}(s) d s-\int_{\left[t^{*},\left(t^{*}+s\right)\right]_{\mathrm{imp}}^{\sigma}} \tilde{\Lambda} d s\right) \\
& \leq \exp \left(\int_{\left[t^{*}+s, t^{*}\right]_{\mathrm{avg}}^{\sigma}} \mu d s+\int_{\left[t^{*}+s, t^{*}\right]_{\mathrm{imp}}^{\sigma}} \Lambda d s\right) \\
& \leq \exp (\mu r),
\end{aligned}
$$

where in the last inequality the fact that $\Lambda \leq \mu$ (implied by the theorem conditions) is used. Following the same argument as in the proof of Theorem 3.1. one can draw a contradiction by applying Itô's formula and the Razumikhin-type argument. Therefore, we have shown that the claim holds on $\left[t_{m}, t_{m+1}\right)$.

(b) $\sigma\left(t_{m}\right) \in \mathcal{N}_{\text {imp }}$. On $\left[t_{m}-r, t_{m}\right)$, one has, by the inductive assumption,

$$
v(t) \leq u(t) \leq C_{m} e^{\mu r},
$$

where

$$
C_{m}=M\left\|v_{t_{0}}\right\| \prod_{i \in \mathcal{N}_{\mathrm{avg}}} \rho_{i}^{N_{\sigma}\left(t_{m}, t_{0} ;\{i\}\right)} \prod_{1 \leq k \leq m} d\left(t_{k}\right) \exp \left(\int_{\left[t_{0}, t_{m}\right]_{\mathrm{avg}}^{\sigma}} \tilde{\mu}_{\sigma(s)}(s) d s-\int_{\left[t_{0}, t_{m}\right]_{\mathrm{imp}}^{\sigma}} \tilde{\Lambda} d s\right)
$$

and

$$
v\left(t_{m}\right)<C_{m} e^{-\bar{\nu}_{i_{m}} \delta_{i_{m}}-\tilde{\Lambda} \delta_{i_{m}}-\varepsilon}<C_{m} e^{-\bar{\nu}_{i_{m}} \delta_{i_{m}}-\tilde{\Lambda} \delta_{i_{m}}}
$$

where $i_{m}=\sigma\left(t_{m}\right)$ and $\varepsilon>0$ is a sufficiently small number.

We shall show that

$$
v(t) \leq C_{m} e^{-\tilde{\Lambda} \delta_{i_{m}}}, \quad \forall t \in\left[t_{m}, t_{m+1}\right) .
$$

Assume (3.30) is not true. Define

$$
\bar{t}=\inf \left\{t \in\left[t_{m}, t_{m+1}\right): v(t)>C_{m} e^{-\tilde{\Lambda} \delta_{i_{m}}}\right\} .
$$

and

$$
\underline{t}=\sup \left\{t \in\left[t_{m}, \bar{t}\right): v(t) \leq C_{m} e^{-\bar{\nu}_{i_{m}} \delta_{i_{m}}-\tilde{\Lambda} \delta_{i_{m}}-\varepsilon}\right\}
$$

Then

$$
v(\underline{t})=C_{m} e^{-\bar{\nu}_{i_{m}} \delta_{i_{m}}-\tilde{\Lambda} \delta_{i_{m}}-\varepsilon}=e^{-\bar{\nu}_{i_{m}} \delta_{i_{m}}-\varepsilon} v(\bar{t}),
$$

and

$$
\begin{array}{ll}
v(t) \leq v(\bar{t}), \quad \forall t \in\left[t_{m}, \bar{t}\right], \\
v(t) \geq v(\underline{t}), \quad \forall t \in[\underline{t}, \bar{t}] .
\end{array}
$$

Therefore, for $t \in[\underline{t}, \bar{t}]$ and $s \in[-r, 0]$, from (3.29), (3.32), and (3.33), one can obtain

$$
v(t+s) \leq C_{m} e^{\mu r} \leq e^{\mu r+\bar{\nu}_{i_{m}} \delta_{i_{m}}+\tilde{\Lambda} \delta_{i_{m}}+\varepsilon} v(\underline{t}) \leq q_{i_{m}} v(t),
$$

provided that $\varepsilon>0$ has been chosen sufficiently small. 
By the Razumikhin-type conditions (iii) and applying Itô's formula on $[\underline{t}, \bar{t}]$, one can show that

$$
v(\bar{t}) \leq v(\underline{t}) e^{\bar{\nu}_{i_{m}} \delta_{i_{m}}},
$$

which would contradict (3.31). Therefore (3.30) must be true. It follows immediately that the claim holds on $\left[t_{m}, t_{m+1}\right)$.

We can now conclude that the claim holds for all $t \geq t_{0}$. By the arbitrary choice of $\eta$, we actually have

$v(t) \leq M\left\|v_{t_{0}}\right\| \prod_{i \in \mathcal{N}_{\text {avg }}} \rho^{N_{\sigma}\left(t, t_{0} ;\{i\}\right)} \prod_{t_{0}<t_{k} \leq t} d\left(t_{k}\right) \exp \left(\int_{\left[t_{0}, t\right]_{\text {avg }}^{\sigma}} \mu_{\sigma(s)}(s) d s-\int_{\left[t_{0}, t_{\sigma}\right]_{\mathrm{imp}}^{\sigma}} \Lambda d s\right)$,

which, by the fact that $\sigma \in \mathcal{I}_{\mathcal{S}}$, implies

$$
\begin{aligned}
v(t) & \leq M \hat{d}\left\|v_{t_{0}}\right\| \prod_{i \in \mathcal{N}_{\text {avg }}} \rho_{i}^{N_{0}} \exp \left(\int_{\left[t_{0}, t\right]_{\text {avg }}^{\sigma}}\left[\frac{\ln \left(\rho_{\sigma(s)}\right)}{\tau_{\sigma(s)}}-\lambda_{\sigma(s)}\right] d s-\int_{\left[t_{0}, t_{\sigma}\right]_{\mathrm{imp}}^{\sigma}} \Lambda d s\right) \\
& =M \hat{d}\left\|v_{t_{0}}\right\| \prod_{i \in \mathcal{N}_{\mathrm{avg}}} \rho_{i}^{N_{0}} e^{-\Lambda\left(t-t_{0}\right)} .
\end{aligned}
$$

Finally, by (3.1), we have

$$
\mathbb{E}\left(|x(t)|^{p}\right) \leq C \mathbb{E}\left(\|\xi\|^{p}\right) e^{-\Lambda\left(t-t_{0}\right)}, \quad \forall t \geq t_{0},
$$

with $C=M \hat{d} \prod_{i \in \mathcal{N}_{\text {avg }}} \rho_{i}^{N_{0}} c_{2} / c_{1}$, which shows the $p$ th moment of system (2.1) is g.u.e.s. with its $p$ th moment Lyapunov exponent not greater than $-\Lambda$.

\section{Almost Sure Exponential Stability}

In this section, with some additional conditions, we show that the trivial solution of system (2.1) is also almost surely exponential stable, provided that the conditions in Theorems 3.1, 3.2, or 3.3 are satisfied.

Theorem 4.1. Let $p \geq 1, C>0$ and $\Lambda>0$. Suppose there exists a constant $K>0$ such that

$$
\mathbb{E}\left(\left|f_{i}(t, \phi)\right|^{p} \vee\left|g_{i}(t, \phi)\right|^{p} \vee|I(i, j, t, \phi)|^{p}\right) \leq K \sup _{-r \leq s \leq 0} \mathbb{E}\left(|\phi(s)|^{p}\right),
$$

for all $i, j \in \mathcal{N}$ and $(t, \phi) \in \mathcal{L}_{\mathcal{F}_{t}}^{p}$. Moreover, suppose $(\sigma, I) \in \mathcal{I}_{\mathcal{S}}^{\mathcal{A}}\left(\tau, N_{0} ; \mathcal{N}\right)$. Then

$$
\mathbb{E}\left(|x(t ; \xi, \sigma, I)|^{p}\right) \leq C \mathbb{E}\left(\|\xi\|^{p}\right) e^{-\Lambda\left(t-t_{0}\right)}, \quad t \geq t_{0},
$$

implies

$$
\limsup _{t \rightarrow \infty} \frac{1}{t} \log |x(t ; \xi, \sigma, I)| \leq-\frac{\Lambda}{p}, \quad \text { a.s. }
$$

where $x(t ; \xi, \sigma, I)$ is a given solution to system (2.1).

Proof. Write $x(t)=x(t ; \xi, \sigma, I)$ in the following. Let $s_{m}=t_{0}+m r$, where $m \geq 1$ is a positive integer. Consider system (2.1) on $\left[s_{m-1}, s_{m}\right]$ for $m \geq 2$. We have

$$
x(t)=x\left(s_{m-1}\right)+\int_{s_{m-1}}^{s_{m}} f_{\sigma(s)}\left(s, x_{s}\right) d s+\int_{s_{m-1}}^{s_{m}} g_{\sigma(s)}\left(s, x_{s}\right) d W(s)+\Sigma_{m}
$$


where

$$
\Sigma_{m}=\sum_{\substack{t_{k} \in \varpi(\sigma, I) \\ t_{k} \in\left(s_{m-1}, s_{m}\right]}} I\left(\sigma\left(t_{k}^{-}\right), \sigma\left(t_{k}\right), t_{k}, x_{t_{k}^{-}}\right)
$$

By Hölder's inequality, 4.4 implies

$$
\begin{aligned}
\mathbb{E}\left(\left\|x_{s_{m}}\right\|^{p}\right) \leq 4^{p-1}[ & \mathbb{E}\left(\left|x\left(s_{m-1}\right)\right|^{p}\right)+\mathbb{E}\left(\int_{s_{m-1}}^{s_{m}}\left|f_{\sigma(s)}\left(s, x_{s}\right)\right| d s\right)^{p} \\
& \left.+\mathbb{E}\left(\sup _{0 \leq h \leq r}\left|\int_{s_{m-1}}^{s_{m-1}+h} g_{\sigma(s)}\left(s, x_{s}\right) d W(s)\right|^{p}\right)+\mathbb{E}\left(\left|\Sigma_{m}\right|^{p}\right)\right] .
\end{aligned}
$$

Now, according to 4.1 and 4.2 and using Hölder's inequality,

$$
\begin{aligned}
\mathbb{E}\left(\int_{s_{m-1}}^{s_{m}}\left|f_{\sigma(s)}\left(s, x_{s}\right)\right| d s\right)^{p} & \leq K r^{p-1} \int_{s_{m-1}}^{s_{m}} \sup _{-r \leq \theta \leq 0} \mathbb{E}\left(|x(s+\theta)|^{p}\right) d s \\
& \leq K r^{p-1} C \mathbb{E}\left(\|\xi\|^{p}\right) \int_{s_{m-1}}^{s_{m}} e^{-\Lambda\left(s-r-t_{0}\right)} d s \\
& \leq K r^{p} C \mathbb{E}\left(\|\xi\|^{p}\right) e^{-\Lambda(m-2) r} .
\end{aligned}
$$

By the Burkholder-Davis-Gundy inequality (see, e.g., [20, p.40, Theorem 7.3]),

$$
\mathbb{E}\left(\sup _{0 \leq h \leq r}\left|\int_{s_{m-1}}^{s_{m-1}+h} g_{\sigma(s)}\left(s, x_{s}\right) d W(s)\right|^{p}\right) \leq C_{p} \mathbb{E}\left(\int_{s_{m-1}}^{s_{m}}\left|g_{\sigma(s)}\left(s, x_{s}\right)\right|^{2} d s\right)^{\frac{p}{2}}
$$

where $C_{p}$ is a constant that depends only on $p$. Note that (4.1) actually implies $\left|g_{i}(t, \phi)\right|^{p} \leq K\|\phi\|^{p}$, for all $(i, t, \phi) \in \mathcal{N} \times\left[t_{0}, \infty\right) \times \mathcal{P C}$. Hence we can show that

$$
\begin{aligned}
& \mathbb{E}\left(\int_{s_{m-1}}^{s_{m}}\left|g_{\sigma(s)}\left(s, x_{s}\right)\right|^{2} d s\right)^{\frac{p}{2}} \\
& \quad \leq \mathbb{E}\left(\sup _{s_{m-1} \leq s \leq s_{m}}\left|g_{\sigma(s)}\left(s, x_{s}\right)\right| \int_{s_{m-1}}^{s_{m}}\left|g_{\sigma(s)}\left(s, x_{s}\right)\right| d s\right)^{\frac{p}{2}} \\
& \quad \leq \kappa \mathbb{E}\left(\sup _{s_{m-1} \leq s \leq s_{m}}\left|g_{\sigma(s)}\left(s, x_{s}\right)\right|^{p}\right)+\kappa^{-1} \mathbb{E}\left(\int_{s_{m-1}}^{s_{m}}\left|g_{\sigma(s)}\left(s, x_{s}\right)\right| d s\right)^{p} \\
& \quad \leq \kappa K \mathbb{E}\left(\sup _{s_{m-1} \leq s \leq s_{m}}\left\|x_{s}\right\|^{p}\right)+\kappa^{-1} r^{p-1} \int_{s_{m-1}}^{s_{m}} \mathbb{E}\left(\left|g_{\sigma(s)}\left(s, x_{s}\right)\right|^{p}\right) d s \\
& \quad \leq \kappa K \mathbb{E}\left(\left\|x_{s_{m}}\right\|^{p}\right)+\kappa K \mathbb{E}\left(\left\|x_{s_{m-1}}\right\|^{p}\right)+\kappa^{-1} K r^{p} C \mathbb{E}\left(\|\xi\|^{p}\right) e^{-\Lambda(m-2) r},
\end{aligned}
$$


where $\kappa>0$ is to be chosen later. Since $\sigma \in \mathcal{I}_{\mathcal{S}_{a}}\left(\tau, N_{0}\right)$, there are at most $N=\left\lfloor r / \tau+N_{0}\right\rfloor$ terms in $\Sigma_{m}$ and hence

$$
\begin{aligned}
\mathbb{E}\left(\left|\Sigma_{m}\right|^{p}\right) & \leq N^{p-1} \sum_{\substack{t_{k} \in \varpi(\sigma, I) \\
t_{k} \in\left(s_{m-1}, s_{m}\right]}}\left|I\left(\sigma\left(t_{k}^{-}\right), \sigma\left(t_{k}\right), t_{k}, x_{t_{k}^{-}}\right)\right|^{p} \\
& \leq N^{p-1} K \sum_{\substack{t_{k} \in \varpi(\sigma, I) \\
t_{k} \in\left(s_{m-1}, s_{m}\right]}} \sup _{-r \leq s<0} \mathbb{E}\left(\left|x\left(t_{k}+s\right)\right|^{p}\right) \\
& \leq N^{p} K C \mathbb{E}\left(\|\xi\|^{p}\right) e^{-\Lambda(m-2) r} .
\end{aligned}
$$

Combining (4.5)-(4.9) gives

$$
\mathbb{E}\left(\left\|x_{s_{m}}\right\|^{p}\right) \leq 4^{p-1} \kappa K C_{p}\left[\mathbb{E}\left(\left\|x_{s_{m}}\right\|^{p}\right)+\mathbb{E}\left(\left\|x_{s_{m-1}}\right\|^{p}\right)\right]+L e^{-\Lambda(m-2) r},
$$

where $L=\left[\left(C_{p} \kappa^{-1} r^{p}+N^{p}\right) K C+1\right] \mathbb{E}\left(\|\xi\|^{p}\right)$, a quantity independent of $k$. Now choose $\kappa>0$ sufficiently small such that

$$
0<\frac{4^{p-1} \kappa K C_{p}}{1-4^{p-1} \kappa K C_{p}}<e^{-\Lambda r}
$$

Then 4.10 implies

$$
\mathbb{E}\left(\left\|x_{s_{m}}\right\|^{p}\right) \leq e^{-\Lambda r} \mathbb{E}\left(\left\|x_{s_{m-1}}\right\|^{p}\right)+L e^{-\Lambda(m-2) r},
$$

which is valid for all $m \geq 2$ and hence, by induction, implies

$$
\begin{aligned}
\mathbb{E}\left(\left\|x_{s_{m}}\right\|^{p}\right) & \leq e^{-\Lambda(m-1) r} \mathbb{E}\left(\left\|x_{s_{1}}\right\|^{p}\right)+(m-1) L e^{-\Lambda(m-2) r}, \\
& \leq\left[\mathbb{E}\left(\left\|x_{s_{1}}\right\|^{p}\right)+e^{\Lambda r}\right] m L e^{-\Lambda(m-1) r}, \quad \forall m \geq 2 .
\end{aligned}
$$

Define, for $m \geq 2$,

$$
A_{m}:=\left\{\omega:\left\|x_{s_{m}}\right\|^{p}>e^{-(\Lambda-\varepsilon)(m-1) r}\right\},
$$

where $\varepsilon \in(0, \Lambda)$ is arbitrary. By 4.11),

$$
P\left(A_{m}\right) \leq e^{(\Lambda-\varepsilon)(m-1) r} \mathbb{E}\left(\left\|x_{s_{m}}\right\|^{p}\right) \leq\left[\mathbb{E}\left(\left\|x_{s_{1}}\right\|^{p}\right)+e^{\Lambda r}\right] m L e^{-\varepsilon(m-1) r} .
$$

The Borel-Cantelli lemma implies that $P\left(\lim \sup _{m \rightarrow \infty} A_{m}\right)=0$, i.e. for almost all $\omega \in \Omega$, there exists an $M(\omega)$ such that

$$
\left\|x_{s_{m}}\right\|^{p} \leq e^{-(\Lambda-\varepsilon)(m-1) r}, \quad \forall m \geq M(\omega),
$$

which implies that, for $t \in\left[s_{m-1}, s_{m}\right]$,

$$
\frac{1}{t} \log |x(t)|^{p} \leq-\frac{(\Lambda-\varepsilon)(m-1) r}{t_{0}+m r}, \quad \forall m \geq M(\omega) .
$$

Therefore, for all most all $\omega \in \Omega$,

$$
\limsup _{t \rightarrow \infty} \frac{1}{t} \log (|x(t)|) \leq \lim _{m \rightarrow \infty}-\frac{(\Lambda-\varepsilon)(m-1) r}{\left(t_{0}+m r\right) p}=-\frac{\Lambda-\varepsilon}{p} .
$$

Since $\varepsilon \in(0, \Lambda)$ is arbitrary, 4.3) follows from 4.13). The proof is complete. 
Corollary 4.1. If $p \geq 1$, then the same conditions as in Theorems 3.1-3.2 together with the conditions in Theorem 4.1 imply (4.3), i.e. the trivial solution of system (2.1) is also almost surely exponentially stable with its Lyapunov exponent not greater than $-\Lambda / p$.

If $0<p<1$, a slightly stronger assumption on the coefficients $f_{i}$ and $g_{i}$ is needed while the assumption on $I$ remains the same.

Theorem 4.2. Let $0<p<1$ and suppose there exists a constant $K>0$ such that the solution process $x_{t}$ satisfies

$$
\mathbb{E}\left(\sup _{-r \leq s \leq 0}\left[\left|f_{i}\left(t+s, x_{t+s}\right)\right|^{p} \vee\left|g_{i}\left(t+s, x_{t+s}\right)\right|^{p}\right]\right) \leq K \sup _{-2 r \leq s \leq 0} \mathbb{E}\left(|x(s)|^{p}\right),
$$

for all $i \in \mathcal{N}$ and $t \geq t_{0}+r$ and

$$
\mathbb{E}\left(|I(i, j, t, \phi)|^{p}\right) \leq K \sup _{-r \leq s \leq 0} \mathbb{E}\left(|\phi(s)|^{p}\right),
$$

for all $i, j \in \mathcal{N}$ and $(t, \phi) \in \mathcal{L}_{\mathcal{F}_{t}}^{p}$. Suppose, in addition, $(\sigma, I) \in \mathcal{I}_{\mathcal{S}}^{\mathcal{A}}\left(\tau, N_{0} ; \mathcal{N}\right)$. Then (4.2) implies (4.3).

Proof. From (4.4), we have

$$
\begin{aligned}
\mathbb{E}\left(\left\|x_{s_{m}}\right\|^{p}\right) \leq[\mathbb{E}(\mid & \left.\left.x\left(s_{m-1}\right)\right|^{p}\right)+\mathbb{E}\left(\int_{s_{m-1}}^{s_{m}}\left|f_{\sigma(s)}\left(s, x_{s}\right)\right| d s\right)^{p} \\
& \left.+\mathbb{E}\left(\sup _{0 \leq h \leq r}\left|\int_{s_{m-1}}^{s_{m-1}+h} g_{\sigma(s)}\left(s, x_{s}\right) d W(s)\right|^{p}\right)+\mathbb{E}\left(\left|\Sigma_{m}\right|^{p}\right)\right],
\end{aligned}
$$

where the fact that $\left(\sum a_{i}\right)^{p} \leq \sum a_{i}^{p}$, for nonnegative reals $a_{i}$ and $0<p<1$, is used. According to 4.2 and (4.14),

$$
\begin{aligned}
\mathbb{E}\left(\int_{s_{m-1}}^{s_{m}}\left|f_{\sigma(s)}\left(s, x_{s}\right)\right| d s\right)^{p} & \leq r^{p} \mathbb{E}\left(\sup _{s_{m-1} \leq s \leq s_{m}}\left|f_{\sigma(s)}\left(s, x_{s}\right)\right|^{p}\right) \\
& \leq K r^{p} \sup _{s_{m-2} \leq s \leq s_{m}} \mathbb{E}\left(|x(s)|^{p}\right) \\
& \leq K r^{p} C \mathbb{E}\left(\|\xi\|^{p}\right) e^{-\Lambda(m-2) r} .
\end{aligned}
$$

While (4.7) remains valid, (4.14) implies

$$
\begin{aligned}
\mathbb{E}\left(\int_{s_{m-1}}^{s_{m}}\left|g_{\sigma(s)}\left(s, x_{s}\right)\right|^{2} d s\right)^{\frac{p}{2}} & \leq r^{\frac{p}{2}} \mathbb{E}\left(\sup _{s_{m-1} \leq s \leq s_{m}}\left|g_{\sigma(s)}\left(s, x_{s}\right)\right|^{p}\right) \\
& \leq K r^{\frac{p}{2}} C \mathbb{E}\left(\|\xi\|^{p}\right) e^{-\Lambda(m-2) r}
\end{aligned}
$$


On the other hand, according to 4.15,

$$
\begin{aligned}
\mathbb{E}\left(\left|\Sigma_{m}\right|^{p}\right) & \leq \sum_{\substack{t_{k} \in \varpi(\sigma, I) \\
t_{k} \in\left(s_{m-1}, s_{m}\right]}} \mathbb{E}\left(\left|I\left(\sigma\left(t_{k}^{-}\right), \sigma\left(t_{k}\right), t_{k}, x_{t_{k}^{-}}\right)\right|^{p}\right) \\
& \leq K \sum_{\substack{t_{k} \in \varpi(\sigma, I) \\
t_{k} \in\left(s_{m-1}, s_{m}\right]}} \sup _{-r \leq s<0} \mathbb{E}\left(\left|x\left(t_{k}+s\right)\right|^{p}\right) \\
& \leq N K C \mathbb{E}\left(\|\xi\|^{p}\right) e^{-\Lambda(m-2) r} .
\end{aligned}
$$

Combining (4.16)-4.19) gives

$$
\mathbb{E}\left(\left\|x_{s_{m}}\right\|^{p}\right) \leq 4^{p-1} \kappa K C_{p}\left[\mathbb{E}\left(\left\|x_{s_{m}}\right\|^{p}\right)+\mathbb{E}\left(\left\|x_{s_{m-1}}\right\|^{p}\right)\right]+L e^{-\Lambda(m-2) r},
$$

where $L=\left[K\left(r^{p}+C_{p} r^{\frac{p}{2}}+N\right)+1\right] C \mathbb{E}\left(\|\xi\|^{p}\right)$, a quantity independent of $k$. The rest of proof is the same as in the proof of Theorem 4.1.

Remark 4.1. Deriving almost sure exponential stability from exponential stability in moment under certain conditions on the growth of coefficients for stochastic functional differential equation is first done in [19] (see also [20, p.175-178]). The proofs here for Theorem 4.1 and Theorem 4.2 are based on the proofs in [20], now taking switching and impulses into account. It can be seen that additional conditions on switching signal and the impulse function are necessary for the implication.

Corollary 4.2. If $0<p<1$, then the same conditions as in Theorems 3.1-3.3 together with the conditions in Theorem 4.2 imply (4.3), i.e. the trivial solution system (2.1) is also almost surely exponentially stable with its sample Lyapunov exponent not greater than $-\Lambda / p$.

\section{EXAMPLES}

In this section, we shall omit mentioning the initial data, which are always assumed to be in $\mathcal{L}_{\mathcal{F}_{t_{0}}}^{\mathrm{b}}$. For simplicity, only second moment stability $(p=2)$ is considered. Almost sure stability would follow from Theorem 4.1 under suitable assumptions.

Example 5.1. Consider the switched stochastic delay system

$$
\begin{aligned}
& d x(t)=[\left.A_{\sigma} x(t)+f_{\sigma}(t, x(t), x(t-r(t)))\right] d t \\
& \quad+g_{\sigma}(t, x(t), x(t-r(t))) d W(t), \quad t \notin \varpi(\sigma, I), \quad t>t_{0}, \\
& \Delta x(t)=I\left(\sigma\left(t^{-}\right), \sigma(t), t, x_{t^{-}}\right), \quad t \in \varpi(\sigma, I),
\end{aligned}
$$

where $\sigma:[0, \infty) \rightarrow \mathcal{N}$ and there exist positive constants $\alpha_{i}, \tilde{\alpha}_{i}, \beta_{i}$, and $\tilde{\beta}_{i}$ such that

$$
\left.\mid f_{i}(t, x, y)\right)\left|\leq \alpha_{i}\right| x\left|+\tilde{\alpha}_{i}\right| y \mid
$$

and

$$
\left|g_{i}(t, x, y)\right|^{2} \leq \beta_{i}|x|^{2}+\tilde{\beta}_{i}|y|^{2},
$$


for $i \in \mathcal{N}$ and $(t, x, y) \in\left[t_{0}, \infty\right) \times \mathbb{R}^{n} \times \mathbb{R}^{n}$. A single time-varying delay is given by $r(t)$, which is continues on $\left[t_{0}, \infty\right)$ and satisfies $0 \leq r(t) \leq r$, for some constant $r>0$.

Stability analysis. Assume that there exist real symmetric matrices $P_{i}$ and $Q_{i}$ such that $P_{i}$ is positive definite and $A_{i}^{T} P_{i}+P_{i} A_{i}=Q_{i}$, for all $i \in \mathcal{N}$. Let $V_{i}(t, x)=x^{T} P_{i} x, i \in \mathcal{N}$. Then

$$
\min _{i \in \mathcal{N}} \lambda_{\min }\left(P_{i}\right)|x|^{2} \leq V_{i}(t, x) \leq \max _{i \in \mathcal{N}} \lambda_{\max }\left(P_{i}\right)|x|^{2},
$$

for all $(i, t, x) \in \mathcal{N} \times\left[t_{0}-r, \infty\right) \times \mathbb{R}^{n}$. For $\phi \in \mathcal{L}_{\mathcal{F}_{t}}^{2}$, we have

$$
\begin{aligned}
& \mathcal{L} V_{i}(t, \phi)=2 \phi^{T}(0) P_{i}\left[A_{i} \phi(0)+f_{i}(t, \phi, \phi(-r(t)))\right] \\
&+ \operatorname{trace}\left[g_{i}^{T}(t, \phi(0), \phi(-r(t))) P_{i} g_{i}(t, \phi(0), \phi(-r(t)))\right] \\
& \leq \lambda_{\max }\left(Q_{i}\right)|\phi(0)|^{2}+2 \alpha_{i} \lambda_{\max }\left(P_{i}\right)|\phi(0)|^{2} \\
&+2 \tilde{\alpha}_{i} \lambda_{\max }\left(P_{i}\right)|\phi(0) \phi(-r(t))| \\
&+\beta_{i} \lambda_{\max }\left(P_{i}\right)|\phi(0)|^{2}+\tilde{\beta}_{i} \lambda_{\max }\left(P_{i}\right)|\phi(-r(t))|^{2} .
\end{aligned}
$$

If, for some $q>1$, we have

$$
\left.\left.\min _{i \in \mathcal{N}} \mathbb{E}\left(\phi^{T}(s) P_{i} \phi(s)\right)\right) \leq q \mathbb{E}\left(\phi^{T}(0) P_{i} \phi(0)\right)\right), \quad \forall s \in[-r, 0],
$$

which implies,

$$
\left.\min _{i \in \mathcal{N}} \lambda_{\min }\left(P_{i}\right) \mathbb{E}\left(|\phi(s)|^{2}\right) \leq q \mathbb{E}\left(\phi^{T}(0) P_{i} \phi(0)\right)\right), \quad \forall s \in[-r, 0] .
$$

Hence, by (5.4),

$$
\mathbb{E}\left(\mathcal{L} V_{i}(t, \phi)\right) \leq \mu_{i} \mathbb{E}\left(V_{i}(t, \phi(0))\right)
$$

where $\mu_{i}=\kappa_{i}+q \tilde{\kappa}_{i}$, with

$$
\kappa_{i}=\frac{\lambda_{\max }\left(Q_{i}\right)}{\lambda_{\max }\left(P_{i}\right)}+\left(2 \alpha_{i}+\tilde{\alpha}_{i}+\beta_{i}\right) \frac{\lambda_{\max }\left(P_{i}\right)}{\lambda_{\min }\left(P_{i}\right)},
$$

and

$$
\tilde{\kappa}_{i}=\left(\tilde{\alpha}_{i}+\tilde{\beta}_{i}\right) \frac{\lambda_{\max }\left(P_{i}\right)}{\min _{i \in \mathcal{N}} \lambda_{\min }\left(P_{i}\right)} .
$$

\section{Average dwell-time approach.}

If $\kappa_{i}+\tilde{\kappa}_{i}<0$ for all $i \in \mathcal{N}$, it is clear that the equations

$$
-\left(\kappa_{i}+q \tilde{\kappa}_{i}\right)=\frac{\log (q)}{r}
$$

for $i \in \mathcal{N}$, each has a unique solution $q_{i}$ in $\left(1,-\kappa_{i} / \tilde{\kappa}_{i}\right)$. Let $q=\max _{i \in \mathcal{N}} q_{i}$, and $\mu=\max _{i \in \mathcal{N}}\left(-\mu_{i}\right)$. Then $q=e^{\mu r}$ and condition (ii) of Theorem 3.1 is satisfied. Assume, in this case, the impulse function is constantly zero. We have $V_{i}(t, x) \leq \max _{i \in \mathcal{N}} \lambda_{\max }\left(P_{i} P_{j}^{-1}\right) V_{j}(t, x)$, for all $(t, x) \in\left[t_{0}, \infty\right) \times \mathbb{R}^{n}$ and $i, j \in \mathcal{N}$, which implies that condition (b) of Theorem 3.1 is satisfied with $\rho_{i}=\max _{j \in \mathcal{N}} \lambda_{\max }\left(P_{i} P_{j}^{-1}\right)$. By Theorem 3.1, the trivial solution of system 5.1 is second moment globally uniformly exponentially stable on $\bigcap_{i \in \mathcal{N}} \mathcal{I}_{\mathcal{S}}^{\mathcal{A}}\left(\tau_{i}, N_{0} ;\{i\}\right)$, 
where $\tau_{i}>\ln \left(\rho_{i}\right) /\left(-\mu_{i}\right)$, for all $i \in \mathcal{N}$, and its second moment Lyapunov exponent is not greater than $-\Lambda=-\min _{i \in \mathcal{N}}\left\{\left(-\mu_{i}\right)-\ln \left(\rho_{i}\right) / \tau_{i}\right\}$.

\section{Impulsive stabilization.}

If $\kappa_{i}+\tilde{\kappa}_{i} \geq 0$ for all $i \in \mathcal{N}$, we can consider the situation as all subsystems of (5.1) without impulses are unstable and seek impulsive stabilization for (5.1) by Theorem 3.2. For given $\Lambda$ and $r$, we can find $q_{i}>1$ such that

$$
q_{i}=\exp \left(\Lambda r+\Lambda \delta_{i}+\mu_{i} \delta_{i}\right)
$$

where $\mu_{i}=\kappa_{i}+q_{i} \tilde{\kappa}_{i}$, provided that $\delta_{i}$ is sufficiently small. Now according to Theorem 3.2 , if we choose the impulses accordingly such that $\delta_{i}<-\ln \left(\rho_{i}\right) /\left(\Lambda+\mu_{i}\right)$, then the trivial solution of system (5.1) is second moment globally uniformly exponentially stable on $\bigcap_{i \in \mathcal{N}} \mathcal{I}_{\mathcal{S}}^{\mathcal{U}}\left(\delta_{i},\{i\}\right)$ with a second moment Lyapunov exponent not greater than $-\Lambda$.

\section{Combined hybrid strategy.}

Suppose $\mathcal{N}=\mathcal{N}_{\text {avg }} \cup \mathcal{N}_{\text {imp }}$ with $\kappa_{i}+\tilde{\kappa}_{i}<0$, for all $i \in \mathcal{N}_{\text {avg }}$, and $\kappa_{i}+\tilde{\kappa}_{i} \geq 0$, for

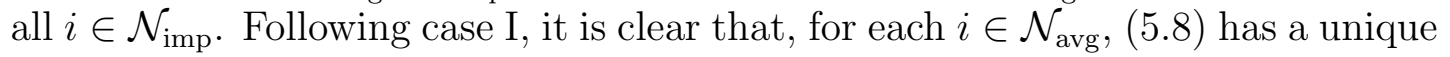
solution $q_{i}$ in $\left(1,-\kappa_{i} / \tilde{\kappa}_{i}\right)$. Let $q=\max _{i \in \mathcal{N}_{\text {avg }}} q_{i}$ and $\mu=\max _{i \in \mathcal{N}_{\text {avg }}}\left(-\mu_{i}\right)$. Then $q=e^{\mu r}$ and condition (ii) of Theorem 3.3 is satisfied. Choose the average dwelltime $\tau_{i}>\ln \left(\rho_{i}\right) /\left(-\mu_{i}\right)$ for $i \in \mathcal{N}_{\text {avg. }}$. We seek appropriate impulsive stabilization for the subsystems in $\mathcal{N}_{\text {imp. }}$. Let $\Lambda=\min _{i \in \mathcal{N}_{\text {avg }}}\left\{\left(-\mu_{i}\right)>\ln \left(\rho_{i}\right) / \tau_{i}\right\}$. For each $i \in \mathcal{N}_{\text {imp }}$ and a sufficiently small $\delta_{i}$, we can find $q_{i}>1$ such that

$$
q_{i}=\exp \left(\mu r+\Lambda \delta_{i}+\mu_{i} \delta_{i}\right),
$$

and $\mu_{i}=\kappa_{i}+q_{i} \tilde{\kappa}_{i}$. According to Theorem 3.3, if we choose the impulses such that $\delta_{i}<-\ln \left(\rho_{i}\right) /\left(\Lambda+\mu_{i}\right)$ for $i \in \mathcal{N}_{\text {imp }}$, then the trivial solution of system (5.1) is second moment globally uniformly exponentially stable on

$$
\left\{\bigcap_{i \in \mathcal{N}_{\text {imp }}} \mathcal{I}_{\mathcal{S}}^{\mathcal{U}}\left(\delta_{i},\{i\}\right)\right\} \bigcap\left\{\bigcap_{i \in \mathcal{N}_{\text {avg }}} \mathcal{I}_{\mathcal{S}}^{\mathcal{A}}\left(\tau, N_{0} ;\{i\}\right)\right\},
$$

with its second moment Lyapunov exponent not greater than $-\Lambda$.

The stability analysis for Example 5.1 can be summarized in the following Theorem, while the notations are explained in the above argument.

Theorem 5.1. The trivial solution of system (5.1) is second moment globally uniformly exponentially stable on

(i) $\bigcap_{i \in \mathcal{N}} \mathcal{I}_{\mathcal{S}}^{\mathcal{A}}\left(\tau_{i}, N_{0} ;\{i\}\right)$, if $\kappa_{i}+\tilde{\kappa}_{i}<0$ and $\tau_{i}>\ln \left(\rho_{i}\right) /\left(-\mu_{i}\right)$, for all $i \in \mathcal{N}$,

(ii) $\bigcap_{i \in \mathcal{N}} \mathcal{I}_{\mathcal{S}}^{\mathcal{U}}\left(\delta_{i},\{i\}\right)$, if $\kappa_{i}+\tilde{\kappa}_{i} \geq 0$ and $\delta_{i}<-\ln \left(\rho_{i}\right) /\left(\Lambda+\mu_{i}\right)$, for all $i \in \mathcal{N}$,

(iii) $\left\{\bigcap_{i \in \mathcal{N}_{\text {imp }}} \mathcal{I}_{\mathcal{S}}^{\mathcal{U}}\left(\delta_{i},\{i\}\right)\right\} \bigcap\left\{\bigcap_{i \in \mathcal{N}_{\text {avg }}} \mathcal{I}_{\mathcal{S}}^{\mathcal{A}}\left(\tau, N_{0} ;\{i\}\right)\right\}$, if $\kappa_{i}+\tilde{\kappa}_{i}<0$ and $\tau_{i}>$ $\ln \left(\rho_{i}\right) /\left(-\mu_{i}\right)$, for all $i \in \mathcal{N}_{\text {avg }}$, and $\kappa_{i}+\tilde{\kappa}_{i} \geq 0$ and $\delta_{i}<-\ln \left(\rho_{i}\right) /\left(\Lambda+\mu_{i}\right)$, for all $i \in \mathcal{N}_{\text {imp }}$.

In all three cases, the second moment Lyapunov exponent is guaranteed to be not greater than $-\Lambda$. If, in addition, assumptions of Theorem 4.1 are satisfied, then the Lyapunov exponent is guaranteed to be not greater than $-\Lambda / 2$. 
Example 5.2. Let $\mathcal{N}=\{1,2\}$ and choose

$$
A_{1}=\left(\begin{array}{ccc}
-4.05 & 2.01 & -1.31 \\
-4.53 & -4.18 & -0.33 \\
-1.24 & 0.15 & -3.15
\end{array}\right), \quad A_{2}=\left(\begin{array}{ccc}
-0.87 & -0.64 & 0.05 \\
0.87 & 0.85 & 2.70 \\
-1.52 & -3.37 & 0.59
\end{array}\right)
$$

Consider a special case of (5.1),

$$
d x(t)=\left[A_{\sigma} x(t)+B_{\sigma} x(t-r)\right] d t+C_{\sigma} x(t-r) d W(t), \quad t>t_{0},
$$

with

$$
I(i, j, t, \phi)=D(i, j) \phi(0)
$$

where $r>0$ is a constant, $B_{i}=C_{i}=I_{3}$ ( $I_{3}$ is the $3 \times 3$ identity matrix), and $D(i, j)$ are $3 \times 3$ constant matrices for $i, j \in \mathcal{N}$.

Combined hybrid stabilization. We shall follow the same notations and argument as in Example 5.1. Choose $V_{1}(t, x)=V_{2}(t, x)=x^{2}$ (i.e. $P_{1}=P_{2}=I_{3}$ ). Taking $f_{i}(t, x, y):=B_{i} y, g_{i}(t, x, y):=C_{i} y$, it is clear that (5.2) and (5.3) are satisfied with $\tilde{\alpha}_{i}=\left\|B_{i}\right\|, \tilde{\beta}_{i}=\left\|C_{i}\right\|^{2}$, and $\alpha_{i}=\beta_{i}=0$. We can compute, from (5.6) and (5.7),

$$
\kappa_{1}=\lambda_{\max }\left(A_{1}^{T}+A_{1}\right)+\left\|B_{1}\right\|=-3.0139, \quad \tilde{\kappa}_{1}=\left\|B_{1}\right\|+\left\|C_{1}\right\|^{2}=2,
$$

and

$$
\kappa_{2}=\lambda_{\max }\left(A_{2}^{T}+A_{2}\right)+\left\|B_{2}\right\|=2.4545, \quad \tilde{\kappa}_{2}=\left\|B_{2}\right\|+\left\|C_{2}\right\|^{2}=2 .
$$

Therefore, $\kappa_{1}+\tilde{\kappa}_{1}<0$ and $\kappa_{2}+\tilde{\kappa}_{2}>0$. Following case (iii) of Theorem 5.1 . we can choose a combined hybrid strategy to stabilize system (5.11). The key steps are to find the average dwell-time condition $\tau_{1}$ for the first mode, and the constants $\delta_{2}$ and $\rho_{2}$, which characterize, respectively, the impulse frequency and impulse strength for the second mode. Since $P_{1}=P_{2}=I_{3}$ and there are no impulses applied when the first mode is to be activated, we have that (3.5) (in condition (b) of Theorem 3.1), for $j=1$, is satisfied with $\rho_{1}=1$ and $d(t) \equiv 1$, which, according to Remark 3.2 , implies that there is essentially no average dwelltime restriction for the first mode. Solving (5.8) for $i=1$ gives $q_{1}=1.3550$ and $\mu_{1}=-0.3038$. Hence $\Lambda=\mu=0.3038$. Choose $\delta_{2}=0.1$. Solving (5.10) for $i=2$ gives $q_{2}=3.8765$. Hence $\mu_{2}=10.2075$. To introduce impulses for the second mode only, let

$$
D(i, 1)=0, \quad D(i, 2)=-0.5 I_{3}, \quad i=1,2 .
$$

Therefore, 3.5 is satisfied with $\rho_{2}=0.25$ and $d(t) \equiv 1$. It is verified that

$$
\delta_{2}=0.1<0.1319=-\frac{\ln \left(\rho_{2}\right)}{\Lambda+\mu_{2}} .
$$

According to Theorem 3.2, the trivial solution of $(5.11)$ is second moment globally uniformly exponentially stable and its second moment Lyapunov exponent is not greater than -0.3038. If, in addition, $(\sigma, I)$ satisfies an overall average dwell-time condition, Theorem 4.1 also guarantees that trivial solution of (5.11) is almost surely exponentially stable and its Lyapunov exponent is not greater than -0.1519 . 

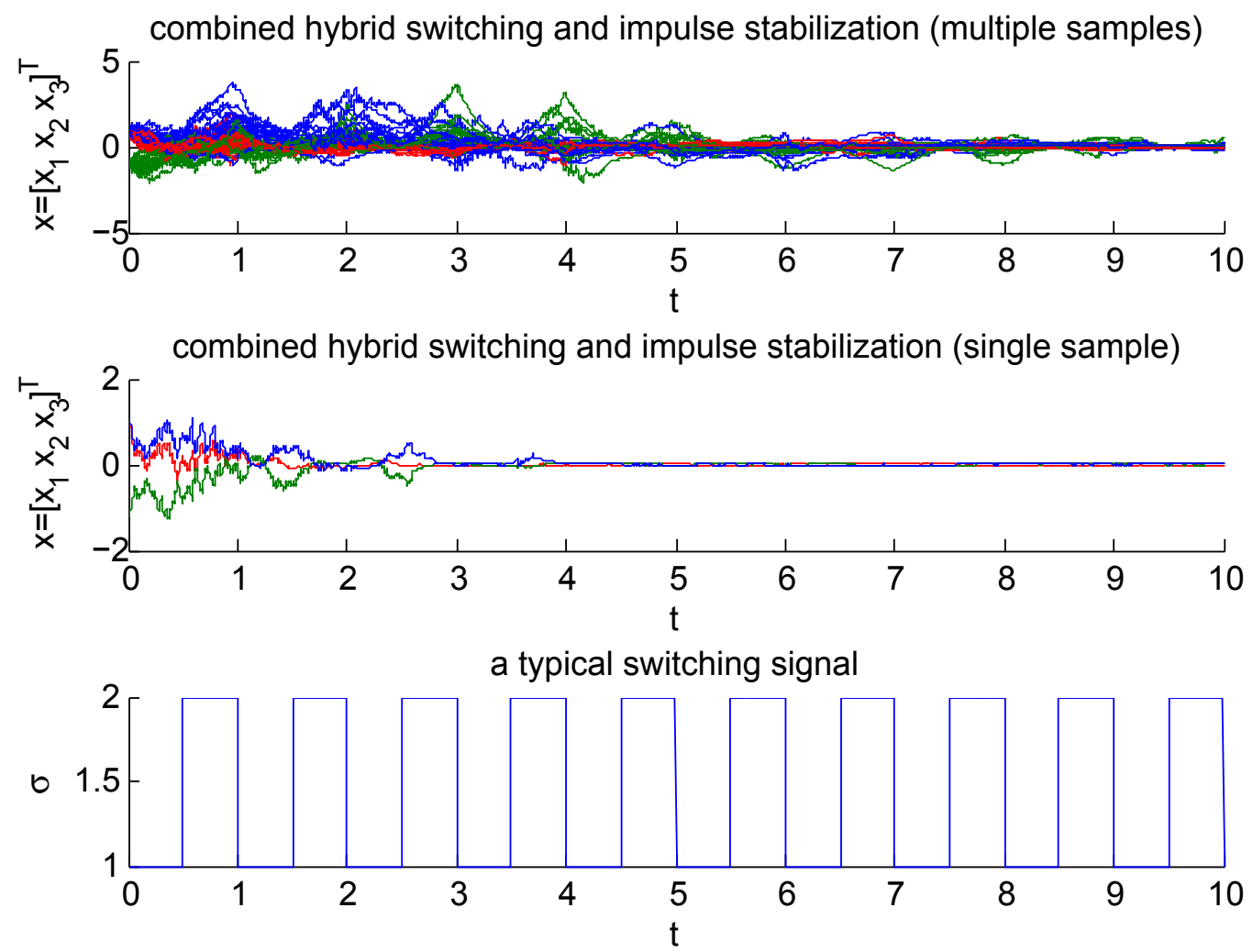

FiguRE 1. Simulation results for Example 5.1.

Numerical simulation. With $(\sigma, I) \in \mathcal{I}_{\mathcal{S}}^{\mathcal{U}}(0.1 ;\{2\})$, typical samples of 5.11 are simulated and shown in Figure 1. It is demonstrated that the combined strategy can successfully stabilize system (5.11), which, in the absence of impulses, can be highly unstable, as shown in Figure 3 .

Average dwell-time approach. Now we illustrate, still by Example 5.2, that Theorem 3.1 can well cover switched systems with both stable and unstable modes and an average dwell-time switching would exponentially stabilize system (5.11). Solving (5.8) for $i=1$ gives $q_{1}=1.3550$ and $\mu_{1}=-0.3038$. Choose $q=q_{1}=$ 1.3550 and $\mu_{2}=\kappa_{2}+q \tilde{\kappa}_{2}=5.1645$. It is easy to verify that condition (ii) of Theorem 3.1 is satisfied. Suppose that there are no impulses, i.e. $D(i, j)=0$ for all $i, j \in \mathcal{N}$. Therefore, (3.5) is satisfied with $\rho_{i}=1$ for $i=1,2$. According to Remark 3.2 , there are no average dwell-time restrictions for both modes. The only condition in 3.1 remains to be verified is condition (c), which reads

$$
\int_{t_{0}}^{t} \mu_{\sigma(s)} d s \leq-\lambda\left(t-t_{0}\right), \quad t \geq t_{0}
$$



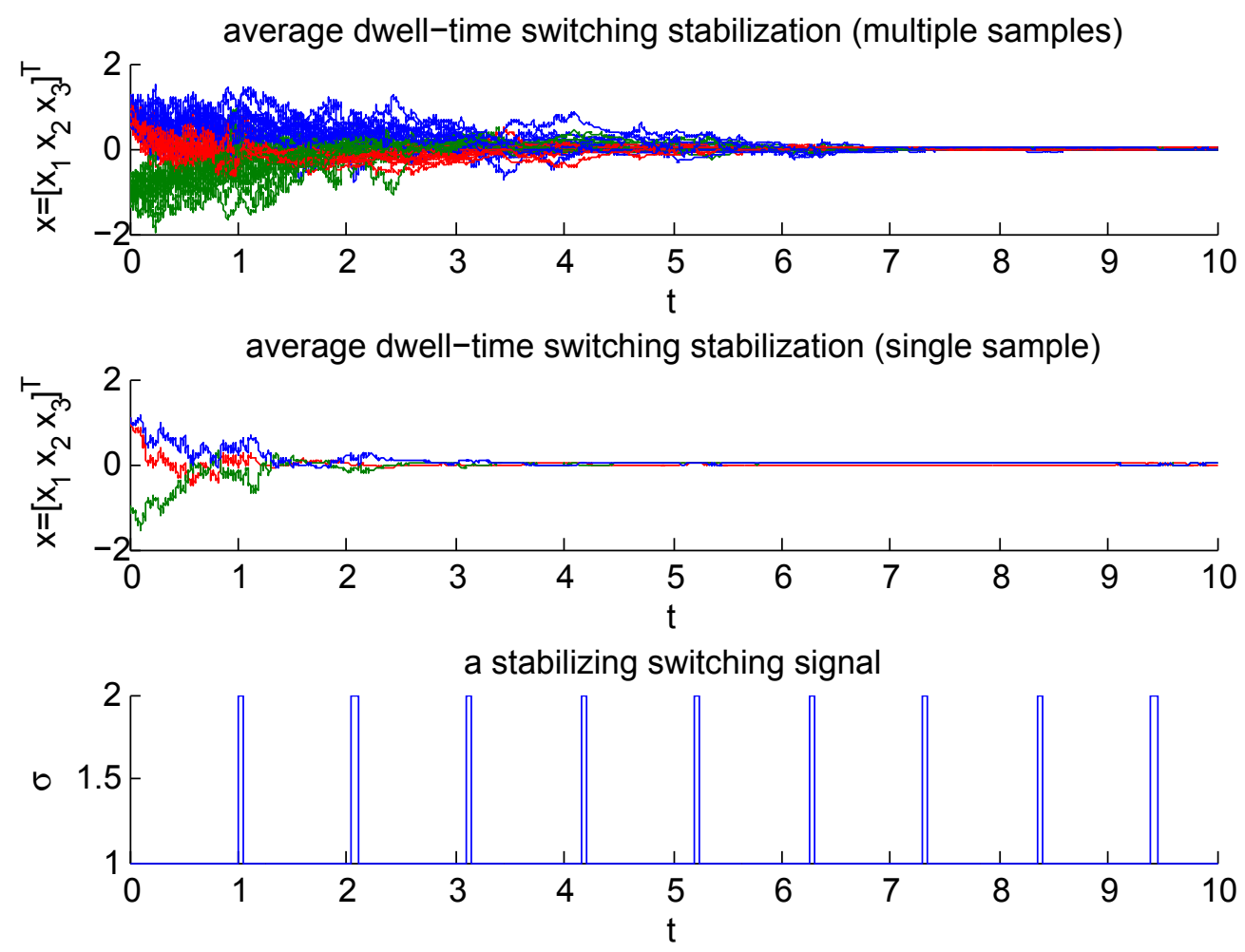

Figure 2. Simulation results for Example 5.1.

for some constant $\lambda>0$. To check (5.13), define, for $i=1,2, \pi_{i}(t)$ to the the total activation time of the $i$ th mode up to time $t$. If $(\sigma, I)$ is such that

$$
\frac{\pi_{2}(t)}{\pi_{1}(t)} \leq \vartheta, \quad t>t_{0} .
$$

for some constant $\vartheta>0$, then, by the identity $\pi_{1}(t)+\pi_{2}(t)=t-t_{0}$, we have

$$
\pi_{1}(t) \geq \frac{t-t_{0}}{1+\vartheta}
$$

and, consequently,

$$
\int_{t_{0}}^{t} \mu_{\sigma(s)} d s=\pi_{1}(t) \mu_{1}+\pi_{2}(t) \mu_{2} \leq \frac{\left(\mu_{1}+\vartheta \mu_{2}\right)\left(t-t_{0}\right)}{1+\vartheta}, \quad t>t_{0} .
$$

Therefore, if we choose $\vartheta=0.05>0$ such that $\mu_{1}+\vartheta \mu_{2}=-0.0456<0$, then (5.13) is verified and Theorem 3.1 guarantees that the trivial solution of (5.11) is second moment globally uniformly exponentially stable and its second moment Lyapunov exponent is not greater than -0.0456. If, in addition, $(\sigma, I)$ satisfies an overall average dwell-time condition, Theorem 4.1 also guarantees that trivial solution of (5.11) is almost surely exponentially stable and its Lyapunov exponent is not greater than -0.0228 . 

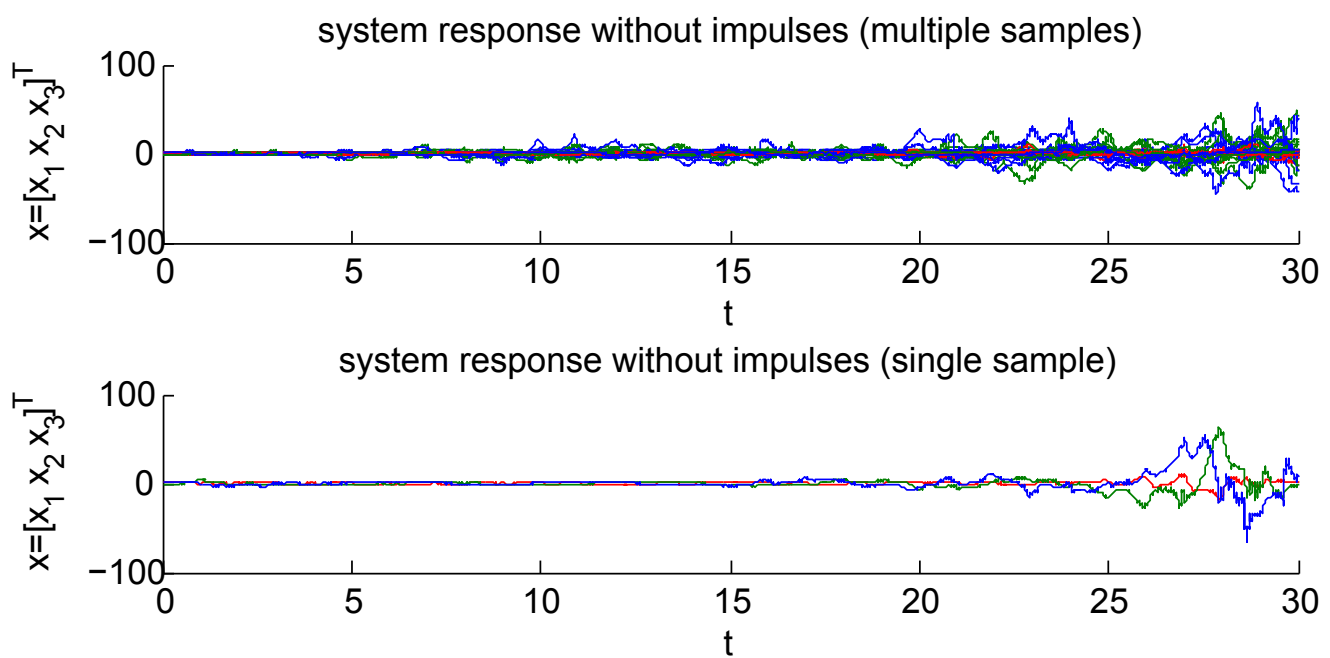

a switching signal that fails to stabilize the system

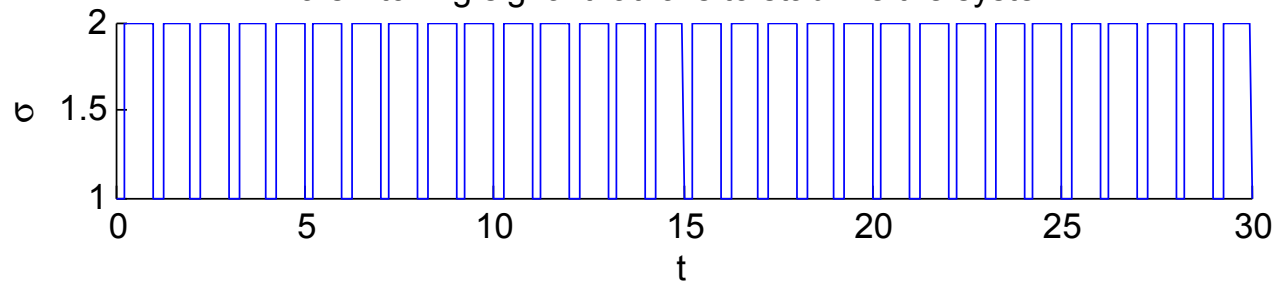

Figure 3. Simulation results for Example 5.1 .

Numerical simulation. With $(\sigma, I)$ satisfying (5.14) with $\vartheta=0.05$, typical samples of (5.11) are simulated and shown in Figure 2, It is demonstrated that the average dwell-time switching under the balance condition (5.14) can successfully stabilize system (5.11), which has both stable and unstable subsystems. Moreover, it is shown in Figure 3 that, a switching signal that fails to satisfy the balance condition (5.14) may also fail to stabilize the system.

Acknowledgements: The research for this work was supported by the Natural Sciences and Engineering Research Council of Canada.

\section{REFERENCES}

1. H. Blom and J. Lygeros (Eds.), Stochastic Hybrid Systems: Theory and Safety Critical Applications, Lecture Notes in Control and Information Sciences, vol. 337, Springer, 2006. 1 .

2. C. Cassandras and J. Lygeros (Eds.), Stochastic Hybrid Systems, Control Engineering Series, vol. 24, Taylor \& Francis CRC Press, 2006. 1

3. O. Diekmann, S. A. van Gils, S. M. V. Lunel, and H.-O. Walther, Delay Equations: Functional-, Complex-, and Nonlinear Analysis, Applied Mathematical Sciences, vol. 110, Springer-Verlag, New York, 1995. 1

4. R. D. Driver, Ordinary and Delay Differential Equations, Applied Mathematical Sciences, vol. 20, Springer-Verlag, New York-Heidelberg, 1977. 1 
5. R. Goebel, R. G. Sanfelice, and A. R. Teel, Hybrid dynamical systems: robust stability and control for systems that combine continuous-time and discrete-time dynamics, IEEE Control Syst. Mag., vol. 29 (2009), 28-93. 1 .

6. J. K. Hale and S. M. V. Lunel, Introduction to Functional Differential Equations, SpringerVerlag, New York, 1993. 1

7. J. P. Hespanha, Uniform stability of switched linear systems: Extension of LaSalle's invariance principle, IEEE Trans. Automat. Control, vol. 49 (2004), 470-482. 1. 2.1

8. J. Hespanha and A. Morse, Stability of switched systems with average dwell-time, in Proc. 38th Conf. Decision and Control, Phoenix, AZ, Dec. 1999, 2655-2660. 3.1

9. V. B. Kolmanovskiu and V. R. Nosov, Stability of Functional-Differential Equations, Mathematics in Science and Engineering, vol. 180, Academic Press, London, 1986. 1

10. V. Lakshmikantham, D. Bainov, and P. Simeonov, Theory of Impulsive Differential Equations, World Scientific Publishing, New Jersey, 1989. 1

11. Z. Li, Y. Soh, and C. Wen, Switched and Impulsive Systems: Analysis, Design, and Applications, Lecture Notes in Control and Information Sciences, vol. 313, Springer-Verlag, Berlin, 2005. 1

12. Y. C. Liao, Switching and impulsive control of a reflected diffusion, Appl. Math. Optim., vol. 11 (1984), 153-159. 1

13. D. Liberzon, Switching in Systems and Control, Boston, MA: Birkhauser, 2003. 1

14. J. Liu, X. Liu, and W.-C. Xie, Delay-dependent robust control for uncertain switched systems with time-delay, Nonlinear Anal. Hybrid Syst., vol. 2 (2008), 81-95. 1

15. J. Liu, X. Liu, and W.-C. Xie, Exponential stability of switched stochastic delay systems with nonlinear uncertainties, Internat. J. Systems Sci., vol. 40 (2009), 637-648. 1

16. J. Liu, X. Liu, and W.-C. Xie, Robust stabilization of stochastic switched delay systems via state-dependent switching rule, Dynam. Systems Appl., vol. 19 (2009), 213-240. 1

17. J. Liu, X. Liu, and W.-C. Xie, Existence and uniqueness results for impulsive and switched hybrid stochastic delay systems, Comm. Appl. Nonlinear Anal., vol. 17 (2010), 37-54. 2

18. J. Liu, X. Liu, and W.-C. Xie, Impulsive stabilization of stochastic functional differential equations, Appl. Math. Lett., vol. 24 (2011), 264-269. 1

19. X. Mao, Razumikhin-type theorems on exponential stability of stochastic functionaldifferential equations, Stochastic Process. Appl., vol. 65 (1996), 233-250. 4.1

20. X. Mao, Stochastic Differential Equations and Applications, Horwood Publishing Limited, Chichester, Second Edition, 2007. 1, 2, 4, 4.1

21. X. Mao and C. Yuan, Stochastic Differential Equations with Markovian Switching, Imperial College Press, London, 2006. 1 .

22. S.-E. A. Mohamedd, Stochastic Functional Differential Equations, Longman Scientific and Technical, New York, 1986. 1, 2

23. A. van der Schaft and H. Schumacher, An Introduction to Hybrid Dynamical Systems, Lecture Notes in Control and Information Sciences, vol. 251, Springer-Verlag, London, 2000. 1

24. R. Shorten, F. Wirth, O. Mason, K. Wulff, and C. King, Stability Criteria for Switched and Hybrid Systems, SIAM Review, vol. 49, pp. 543-732, 2007.1

25. G. Xie and L. Wang, Necessary and sufficient conditions for controllability and observability of switched impulsive control systems, IEEE Trans. Automat. Control, vol. 49 (2004), 960966. 1

26. H. Xu, X. Liu, and K. L. Teo, A LMI approach to stability analysis and synthesis of impulsive switched systems with time delays, Nonlinear Anal. Hybrid Syst., vol. 2 (2008), 38-50. 1

27. H. Xu, X. Liu, and K. L. Teo, Delay independent stability criteria of impulsive switched systems with time-invariant delays, Math. Comput. Modelling, vol. 47 (2008), 372-379. 1

28. H. Xu, X. Liu, and K. L. Teo, Robust stabilization of uncertain impulsive switched systems with delayed control, Comput. Math. Appl., vol. 56 (2008), 63-70. 1 
29. G. Zhai, B. Hu, K. Yasuda, A. N. Michel, Stability analysis of switched systems with stable and unstable subsystems: an average dwell time, Internat. J. Systems Sci., vol. 32 (2001), $1055-1061.3 .1$

30. G. Zong, S. Xu, and Y. Wu, Robust $H_{\infty}$ stabilization for uncertain switched impulsive control systems with state delay: an LMI approach. Nonlinear Anal. Hybrid Syst., vol. 2 (2008), 1287-1300. 1

1 Department of Applied Mathematics, University of Waterloo, Waterloo, Ontario N2L 3G1, Canada

E-mail address: j49liu@uwaterloo.ca

2 Department of Applied Mathematics, University of Waterloo, Waterloo, Ontario N2L 3G1, Canada

E-mail address: xzliu@uwaterloo.ca

${ }^{3}$ Department of Civil and Environmental Engineering, University of Waterloo, Waterloo, Ontario N2L 3G1, Canada

E-mail address: xie@uwaterloo.ca 